Pieds-noirs, têtes hautes: Une histoire des sélectionnés olympiques d'Algérie Author(s): Stanislas Frenkiel

Source: French Colonial History, 2020, Vol. 19 (2020), pp. 163-210

Published by: Michigan State University Press

Stable URL: https://www.jstor.org/stable/10.14321/frencolohist.19.2020.0163

JSTOR is a not-for-profit service that helps scholars, researchers, and students discover, use, and build upon a wide range of content in a trusted digital archive. We use information technology and tools to increase productivity and facilitate new forms of scholarship. For more information about JSTOR, please contact support@jstor.org.

Your use of the JSTOR archive indicates your acceptance of the Terms \& Conditions of Use, available at https://about.jstor.org/terms

Michigan State University Press is collaborating with JSTOR to digitize, preserve and extend access to French Colonial History 


\title{
Pieds-noirs, têtes hautes: Une histoire des sélectionnés olympiques d'Algérie
}

\author{
STANISLAS FRENKIEL \\ Université d'Artois
}

This contribution aims to study the sports careers and biographical trajectories of French athletes from Algeria selected to compete at the Summer Olympics from 1948 to 1984. They are part of the ambiguities and turbulences of the twentieth century: decolonization and globalization, deindustrialization and emancipation, democratization and professionalization of sport. Therefore, the article provides an analysis of the construction of sports excellence proposed at the crossroads of the history of French Algerians, elite sport, and Olympism from the colonial period to the postcolonial era. Relying mainly on ten "oral history" interviews with champions who participated in the Olympic Games, we highlight several determinants structuring their itineraries. These include their urban youth in Algerian and French clubs, sports networks during the Algerian War, the role of the French army, their predominantly amateur athletic careers, their Olympic consecration, and their retraining.

Cette contribution vise à reconstruire les carrières sportives et trajectoires biographiques des Français d'Algérie sélectionnés au Jeux Olympiques d'été entre 1948 et 1984. Elles s'inscrivent dans les ambiguïtés et turbulences du XX ${ }^{\text {ème }}$ siècle:

Stanislas Frenkiel est Maître de Conférences, Atelier SHERPAS (URePSSS-ULR 7369), Faculté des Sports et de l'Éducation Physique, Université d'Artois, France, stanislas.frenkiel@univ-artois.fr. 
décolonisation et mondialisation, désindustrialisation et émancipation, démocratisation et professionnalisation du sport. C'est donc une réflexion sur la construction de l'excellence sportive qui est proposée au carrefour de l'histoire des Français d'Algérie, du sport d'élite et de l'olympisme du temps colonial à lère postcoloniale. Sappuyant principalement sur 10 entretiens "récits de vie" avec des champions ayant participé aux Jeux Olympiques, nous mettons en évidence plusieurs déterminants structurant leurs itinéraires. Leur jeunesse en ville dans des clubs algériens et français, les filières sportives dans la Guerre d'Algérie, le rôle de larmée française, leur carrière majoritairement sous le statut amateur, leur consécration olympique et leur reconversion facilitée en font partie.

À la faveur d'un renouvellement des études sur l'histoire coloniale, les acteurs de la colonisation réapparaissent. Longtemps voués au pilori, les colonisateurs n'avaient pas droit de cité chez les historiens anticolonialistes qui s'intéressaient surtout à léveil politique des colonisés et aux sources des révoltes. [...] Le renouvellement des problématiques ou la relecture de certains chercheurs comme Georges Balandier font ressortir la complexité des relations coloniales et l'interdépendance des hommes vivant dans cet univers. ${ }^{1}$

-Valérie Escanglon-Morin, historienne

L a population la plus importante parmi les rapatriés d'Algérie est constituée des européens, communément appelés pieds-noirs à partir de la fin de la Guerre d’Algérie. L'historienne Joëlle Hureau présente ce terme à la genèse mystérieuse:

Diverses étymologies sont avancées. Le port des bottes ou de chaussures noires par les conquérants français ont justifié la naissance du sobriquet dès 1830. À moins que, plus tardivement, le foulage du raisin teintant les pieds de jus sombre engendre l'image. On indique par ailleurs une possible confusion entre les locutions arabes "homme noir," allusion à l'habit courant au XIX ${ }^{\text {ème }}$ siècle, et "pied noir," phonétiquement très proches. ${ }^{2}$

L’emploi de cette formule pourrait aussi provenir d'une bande de jeunes européens, engagés contre l'Indépendance, du quartier du Ma’arif à Casablanca. L'expression passerait ensuite du Maroc à l'Algérie durant la guerre, employée de manière péjorative en 1962, puis revendiquée comme élément identitaire 
en France où ils sont mal accueillis et rejetés dans le camp des oppresseurs. Près d'un million de pieds-noirs forme un groupe soudé par l'expérience de l'exil malgré leur diversité sociale, religieuse et politique. ${ }^{3}$ L'histoire de ces représentants d'une colonisation ambiguë, à la fois productrice de richesses et profondément inégalitaire, débute avec la conquête et la prise d'Alger en 1830. Dans les années qui suivent et jusquà la Seconde Guerre mondiale, une population composite quittant leur patrie pour des raisons politiques, sociales et économiques s'installe en Algérie.

L'historien Michel Winock rappelle que "la France encourage l'immigration, en provenance soit de la métropole (Alsace-Lorraine, surtout les départements du Midi), soit des pays méditerranéens (Espagne, Italie et Malte). La confiscation, l'expropriation des terres arabes par le contingentement, la colonisation étatique et la colonisation privée permettent d’offrir aux nouveaux colons des lots individuels gratuits ou à bas prix." ${ }^{4}$ Face à la population musulmane paupérisée, peu scolarisée, sous-administrée et plus nombreuse, ces nouveaux habitants accèdent à la citoyenneté française grâce au décret Crémieux de 1870 pour les Algériens de confession israélite et la loi sur la nationalité de 1889 consacrée aux enfants d'étrangers. Sous la Troisième République, le lobby algérien ayant des velléités de séparatisme empêche toute réforme allant dans le sens de l'émancipation, y compris par l'assimilation des populations musulmanes. Se comparant à leurs concitoyens de métropole, "les Français d’Algérie ne perçoivent pas qu’ils sont partie prenante du fait colonial, c'est-à-dire d'un système de privilèges dont ils bénéficient par rapport aux colonisés." 5 Pour autant, tandis qu'une minorité de colons accumule de considérables fortunes sur la base de terres spoliées avec le concours de l'État, le train de vie de la plupart des Français d'Algérie est inférieur à celui des Français de métropole. Citadins attachés à l'Algérie qu'ils considèrent comme leur pays et le prolongement de la France, ils partagent parfois les mêmes réalités sociales et spatiales que les colonisés. En cela, ils composent une société complète, avec tous les métiers, tous les statuts possibles, et non une caste de riches et d'exploiteurs. Les images de cette population en train de fusionner se transforment en trois phases: médiocre de 1830 à 1880 (pauvreté, dureté du travail, conditions dangereuses et précaires de l'installation et maladies), laudative de 1880 à 1939 (courage, ardeur, capacité d'initiative, invention et génie) et, après une quinzaine d'années d'indifférence, dépréciative de 1954 à nos jours (richesse, égoïsme, exploitation, ignorance, arrogance, pensée réactionnaire et racisme). Afin de répondre à l'invitation lancée par l'historienne Jeannine Verdès-Leroux, 
réévaluer de manière équitable le passé de ces "humiliés de l'histoire," de nouvelles approches doivent être envisagées. ${ }^{6}$

Puisque les activités physiques et sportives se révèlent un extraordinaire analyseur des sociétés où elles se déploient, l'exploration des liens entre les Français d'Algérie, le sport d'élite et l'olympisme du temps colonial à l'ère postcoloniale ${ }^{8}$ peut s'avérer fructueuse. En effet, en France et en Algérie comme ailleurs, les exercices du corps tiennent à toutes les époques une place déterminante dans la construction des sociabilités, des identités et des représentations des groupes, classes et sociétés qui les pratiquent. ${ }^{9}$ Face à l'oubli, au ressentiment et au déchirement, face à la réécriture nostalgique du passé, cette histoire des sélectionnés olympiques d'Algérie jette un regard neuf sur les relations sportives, politiques, culturelles et économiques de part et d’autre de la Méditerranée. Elle contribue aussi à la connaissance de l'évolution contemporaine de ces deux pays, des transferts culturels et du sport de haut-niveau.

En une quarantaine d'années, faisant fi de l'indifférence voire du mépris des élites et de l'anti-intellectualisme de certains journalistes, l'histoire du sport a atteint lâge de sa majorité en France. Des spécialistes font des pratiques, du loisir et du spectacle sportif des objets légitimes. ${ }^{10}$ Les conditions socio-historiques de naissance et de diffusion du sport aux XIX ${ }^{\text {ème }}$ et XX ${ }^{\text {ème }}$ siècles, ${ }^{11}$ ses liens avec la politique, ${ }^{12}$ la persistance de discriminations sportives ${ }^{13}$ et du dopage, ${ }^{14}$ l'importance des acteurs sportifs ${ }^{15}$ et des supporters, ${ }^{16}$ les dérives du sport ${ }^{17}$ sont désormais bien connus. De plus, l'héroïsme et le nationalisme sportif font l'objet de nombreuses études. ${ }^{18}$ Spécifiques au mouvement olympique, les écrits des historiens Allen Guttmann et Patrick Clastres sur la genèse de l'olympisme, ${ }^{19}$ ceux de François Jéquier, Pierre Milza et Philippe Tétart sur ses enjeux politiques, ${ }^{20}$ ceux de l'historienne Sandra Heck sur les prémices du pentathlon moderne ${ }^{21}$ et ceux des politologues Jean-Loup Chappelet et Brenda Kübbler-Mabbott sur le système et le mythe olympique ${ }^{22}$ font référence. Ils s'ajoutent à d'autres travaux sur l'histoire et la philosophie du corps ainsi que les Jeux Paralympiques et ses élites. ${ }^{23}$

Dans un tel contexte, les écrits de l'historien Didier Rey sur le football en Oranie coloniale et ceux du politologue Youssef Fatès sur les liens entre sport et politique en Algérie constituent de précieuses ressources. ${ }^{24}$ Avec d'autres chercheurs, ils explorent l'implantation du basket-ball, du cyclisme et des sports d'hiver en Algérie. ${ }^{25}$ Les récits des journalistes sur le sport algérien étant teintés de tonalités hagiographiques dans chacune des communautés, ${ }^{26}$ d'évidents manques surgissent. Aussi surprenant soit-il, par désintérêt, manque de temps ou difficulté 
supposée d'accéder aux sources, aucun auteur n’analyse la contribution des Français d'Algérie à l'Équipe de France olympique depuis les trois médailles d'or du cycliste de Mostaganem Paul Masson aux Jeux d'Athènes en 1896. Et pourtant, cette histoire pourrait raconter une page originale de l'histoire de l'immigration, de la colonisation et des rapatriés d'Algérie. Nous nous focalisons donc sur les sportifs français nés en Algérie jusqu’en 1962 et sélectionnés aux Jeux Olympiques d'été à partir d'une reconstitution des parcours de vie. ${ }^{27}$ L'appartenance à l'Équipe de France de ces sportifs de différentes générations, parfois multimédaillés, les fait naturellement s'inscrire dans l'élite. Plusieurs questions se posent: qui sont ces sportifs de haut-niveau? Existe-t-il des conditions socioculturelles menant à l'acquisition de dispositions propices à l'engagement dans le sport? Dans les trajectoires des acteurs, dans quelles circonstances découvrent-ils le sport en Algérie et en France ${ }^{28}$ Existe-t-il des ruptures biographiques repérables et récurrentes? Comment se façonne leur excellence sportive au quotidien face à l'exode? Il s'agira non seulement de renforcer les connaissances sur le rôle puissant et contesté du sport comme vecteur d'intégration sociale mais aussi de mener une réflexion sur le capital sportif. ${ }^{29}$ Pendant ou après la carrière, la gestion de cette ressource spécifique (titres sportifs et notoriété, composantes symboliques), produite par le monde sportif et potentiellement transférable dans d'autres régions de l'espace social, y est cruciale d'autant plus qu'elle se dévalue dans le temps. ${ }^{\circ}$

\section{Sources et méthodologie}

Après la constitution d'un cadre théorique international, l'identification de ces 47 Français d’Algérie sélectionnés olympiques se réalise notamment dans les guides de l'Équipe de France olympique édités tous les quatre ans et archivés au Comité National Olympique et Sportif Français à Paris. ${ }^{31}$ La reconstruction de la complexité, manifeste ou voilée, de leurs parcours implique de relever un défi empirique. Pour aboutir à une prosopographie ou biographie collective de ces sportifs, cette contribution repose sur des sources orales et écrites recueillies et analysées thématiquement entre 2008 et 2019. Les sources orales se composent d'entretiens type "récits de vie" menés en France (Aix-en-Provence, Marmande, Marseille, Rocquencourt, Sainte-Maxime et Venelles) auprès de 10 sportifs (voir tableau 1) en utilisant systématiquement un seul guide d'entretien. ${ }^{32}$ Grâce à un pacte biographique ${ }^{33}$ scellé entre le chercheur et l'interviewé, cette approche 
met en rapport plusieurs témoignages sur la même expérience et permet ainsi de "dépasser leurs singularités pour atteindre, par construction progressive, une représentation sociologique des composantes sociales (collectives) de la situation." ${ }^{34}$ Néanmoins, nous ne prétendons pas ici atteindre une quelconque approximation statistique étant donné le nombre limité de témoignages, rapportés avec pudeur qu'ils soient dramatiques ou empreints d'une simple banalité. Il s'agit beaucoup plus de comprendre et de recouper des trajectoires susceptibles d'offrir, par une approche compréhensive, des indications sur les dispositions spécifiques de ces sportifs et d'éventuelles singularités. Le caractère approfondi des entretiens autorise également à préciser la trame institutionnelle sur laquelle se sont construites ces trajectoires. Cela permet de mieux comprendre les sociabilités spécifiques qui ont animé ces sportifs issus de l'Algérie Française.

Dans cette perspective, pour identifier des réseaux d'appartenance et des engagements communs, la difficulté de l'aposteriori causée par la plasticité de la mémoire, la relation d'entretien ${ }^{35}$ et l'illusion biographique ${ }^{36}$ sont prises en compte. Des dizaines de relances téléphoniques restent sans réponse. Un refus catégorique essuyé contraste avec l'hospitalité sincère des champions. Cela nous amène à recouper autant que possible les informations livrées par les acteurs et leurs archives privées en utilisant des sources écrites, parmi lesquelles un ouvrage de référence évoquant Alfred Nakache, ${ }^{37}$ le nageur d'Auschwitz, les biographies du volleyeur Alain Fabiani, ${ }^{38}$ des footballeurs Jean-François Larios ${ }^{39}$ et Christian Lopez, ${ }^{40}$ de l'entraîneur du boxeur Jean-Paul Anton ${ }^{41}$ et du promoteur de boxe Michel Acariès ${ }^{42}$ sans compter un long article sur le footballeur Alexandre Villaplane ${ }^{43}$ et un autre consacré au nageur Joseph Bernardo. ${ }^{44}$ L'intégralité des articles traitant de sport dans la revue associative L’Algérianiste, publiée trimestriellement depuis 1975 à plus de 10 ooo exemplaires par le Cercle Algérianiste, est analysée. Les rares archives audiovisuelles de l'Institut National de l'Audiovisuel (INA) concernant spécifiquement les 10 sportifs interrogés sont consultées ainsi que le documentaire de Jean-Claude Huisman, Algérie, terre de champions, diffusé pour la première fois en 1950.45

Pour relier les expériences vécues et comprendre les changements, l'ambition de cette mise en récit est de neepouser ni "l'histoire officielle" accusée d'épouser la posture des "vainqueurs" (gaullistes, anticolonialistes et "porteurs de valise") ni en réaction la "contre-histoire" victimaire de militants associatifs pieds-noirs qui serait naturellement authentique car vécue de l'intérieur. ${ }^{46} \mathrm{Il}$ ne s'agit pas non plus de succomber aux délices des fausses évidences. La récriture nostalgique de 
l'histoire faisant du sport algérien un élément idyllique d'un métissage culturel témoignant d'une certaine harmonie interethnique de l'Algérie coloniale aurait été un piège. Volontairement, pour lever le voile de l'oubli, une place importante est consacrée à la Guerre d'Algérie dont les plaies saignent encore aujourd'hui. ${ }^{47}$

\section{La production coloniale d'une élite sportive}

Dès la seconde moitié du XIX ${ }^{\text {me }}$ siècle, le sport est pratiqué sur le sol français par des Anglais venus pour leurs affaires et leurs loisirs à Paris et dans certaines stations balnéaires de la Mer du Nord, de la Manche et de locéan Atlantique. Ces premiers sportsmen s'adonnent à l'aviron, la course à pied, le lawn-tennis, le cyclisme, le golf, le tir au pigeon, le hippisme et le skating. Les clubs de football-rugby apparaissent lorsque la communauté britannique est localement suffisamment développée, à l'instar du Havre Athletic Club en 1872.48 Dans le cas algérien, premier territoire colonial touché par le sport, l'hypothèse de cette greffe britannique est sujette à caution. Si de jeunes gens ont pu s'approprier le football en imitant les marins et touristes étrangers, il est fort probable que l'occupation coloniale par la métropole et sa politique de peuplement jouent un rôle prédominant. Comme les gymnastiques commerciales, hygiéniques et patriotiques de l'Union des Sociétés de Gymnastique de France, le sport passe directement d'une rive à l'autre de la Méditerranée, constituant d'abord une activité réservée aux colons. En effet, ils considèrent cette pratique majoritairement masculine comme un signe socialement et racialement distinctif vis-à-vis des “indigènes." Les compétitions sportives entre ces deux groupes sont vigoureusement découragées par les autorités coloniales qui perçoivent le risque d'une revanche symbolique des dominés sur les dominants. ${ }^{49}$ Par exemple, le football apparaît en Algérie à la fin du XIX ${ }^{\text {ème }}$ siècle et se développe rapidement sur une base communautaire, davantage que dans les autres colonies ou protectorats français. À Oran, dans une région soumise à l'emprise coloniale, les turbulents bourgeois membres du Club des Joyeusetés, créé en 1894, revendiquent leurs places de doyens, même s'ils ne pratiquent le football que trois ans plus tard, l'année de fondation du Club Athletic d'Oran..$^{50}$ Principal ciment de la culture coloniale de l'Algérie française, le ballon rond renforce les liens affectifs et institutionnels avec la mère patrie. ${ }^{51} \mathrm{Il}$ fait vibrer l'Algérie coloniale. Malgré la désapprobation des traditionnalistes « vieux turbans » craignant leur dépossession identitaire, 
le charme exotique du football présente un attrait: celui de théâtraliser la lutte entre colonisés et colonisateurs.

Avec l'établissement des ligues, un second indicateur de l'essor du football algérien est lémergence des premiers clubs indigènes. Les principaux acteurs de sa diffusion sont les colons, les militaires, les fonctionnaires, les ecclésiastiques et les instructeurs scolaires qui ne voient pas tous le sport comme un symbole de l'acceptation de la domination coloniale. ${ }^{52} \mathrm{Il}$ est alors fréquent que des musulmans jouent dans des clubs européens; la séparation ethnique ne sera jamais hermétique. Dès les années 1910, de jeunes musulmans, issus des élites urbaines et scolarisées, tentent de former des clubs plutôt que de s'intégrer dans des clubs européens. Ainsi, dès 1921, naît le Mouloudia Club Alger, suivi en 1926 de l'Union Sportive Musulmane Oran et du Club Sportif Constantinois. C’est encore la période du loyalisme. En 1930, les clubs musulmans, placés sous surveillance, se manifestent par leur neutralité. L'historien Paul Dietschy souligne que même si les couleurs du MC Alger sont le vert, couleur de l'Islam, et le rouge, l'une des couleurs préférées du prophète, ses visées sont d'abord récréatives et hygiéniques pour lutter contre l'alcoolisme et les périls vénériens. ${ }^{53}$ Proches du colonat, les rares sportifs musulmans de formation intellectuelle européenne optent pour l'intégration et l'assimilation des valeurs occidentales. ${ }^{54}$ En faisant l'expérience de la solidarité de la vie unioniste, ils découvrent de nouvelles sociabilités. Entre fonctionnaires, notables ou commerçants, les liens qui les unissent aux européens s'opèrent sur la base d'une proximité interpersonnelle plus qu'intercommunautaire.

La tentative de mainmise des autorités coloniales et politiques sur les associations sportives musulmanes remonte aux années 1920. Concevant le sport comme un outil de contrôle et d'acculturation des populations musulmanes, elles ne restent pas insensibles aux incidents de plus en plus nombreux sur les terrains et dans les tribunes. Avec 11 ooo licenciés dans 146 sociétés sportives en 1937 toutes confessions confondues, l'institutionnalisation du football en Algérie est bien lancée. Progressivement, elle s’adosse à la dynamique du nationalisme. Les revendications portent d'abord sur la reconnaissance de la citoyenneté française pour tous. Dans un contexte de mobilisation sociale et d'effervescence politique du Front Populaire, l'échec du projet de Loi Blum-Viollette en 1936 qui prévoit d'accorder la citoyenneté française et le droit de vote aux élites algériennes encourage la création d'autres clubs musulmans comme l'USM Blida. Les incidents se multiplient, particulièrement dans le Constantinois. L'appui de l’armée est autant sollicité que lors des concours hippiques et courses cyclistes. 5 Chaque jour, l’idée 
indépendantiste algérienne gronde plus fort. Chaque saison, les matchs prennent davantage une tournure politique largement passée sous silence par la presse métropolitaine. Les tensions intercommunautaires embrasent le football algérien.

Dès la fin des années 1940, parmi la population musulmane, le football est désormais adopté autant par la bourgeoisie déçue par l'immobilisme colonial que les couches sociales les plus déshéritées. Malgré la fraternisation et l'harmonie interethnique dans quelques clubs, les matchs disputés entre deux équipes locales néchappent plus à la violence. Séparatistes et offensifs face à la puissance coloniale, les nouveaux clubs musulmans comme la Jeunesse Sportive El Biar, le MC Oran et la Jeunesse Sportive de Kabylie deviennent des structures d'encadrement et de formation nationaliste de la jeunesse. Entre les entraînements, leur programme éducatif se veut émancipateur. Il comprend des cours d'histoire, des conférences dispensées par des personnalités politiques et des chants patriotiques ${ }^{56}$ qu'entendent peut-être résonner les boxeurs Omar Kouidri, Marcel Cerdan ${ }^{57}$ et les futurs sélectionnés olympiques de la communauté européenne d’Algérie.

Issus majoritairement de familles peu nombreuses, catholiques et sportives, les futurs sélectionnés olympiques nés entre 1929 et 1961 voient le jour pour la majorité à Alger et Oran ou dans des villes de moyenne importance comme Sétif. À travers leurs histoires surgit celle des pionniers de l'immigration en Algérie depuis la seconde partie du XIX ${ }^{\text {ème }}$ siècle. Elle est d'abord hexagonale venue d'Alsace, d'Aveyron, de Corse et de Savoie mais aussi européenne (Espagne, Hollande, Italie, Suisse et Tchéquie), moyen-orientale (Liban) et mexicaine. Ils grandissent dans des quartiers européens au sein de classes sociales autant diverses que l'est la société européenne d'Algérie des années 1940 et 1950. Si leur mère est souvent au foyer, leur père est agriculteur, boucher, garagiste, militaire ou policier. Certains comme le père du nageur Marc Kamoun, chirurgien à l'hôpital d'Orléansville ${ }^{58}$ puis d'Alger, président du Groupement Sportif Orléansville, occupent des fonctions prestigieuses et rémunératrices. Né en 1929 à Alger, champion de France à 20 ans, Joseph Bernardo raconte son enfance

Merveilleuse. Mon arrière grand-père conduit la diligence entre Alger et Oran. Mon père est ingénieur au Gouvernement Général d’Algérie. Il a une très bonne place. Paralysé de la jambe droite depuis l'enfance, il fait ensuite beaucoup de voile sur son bateau. Il veut que ses enfants pratiquent tous les sports auxquels il n’a pas accès. Je grandis à Alger, en plein centre-ville, dans un milieu européen. L’immeuble de la rue du Général Berthezène nous appartient. On vit dans un très 
grand appartement au quatrième étage. Ma mère me met au monde. Plus tard, on va au cinéma. Avec mon jumeau Bernard, mon père nous emmène souvent à la piscine municipale d'Alger, à Hussein-Dey, pour nous apprendre à nager. Un jour, on tombe sur un de ses amis, Georges Cals, entraineur de la section natation du Racing Universitaire d'Alger. Il s'intéresse à nous. On rejoint le club. Pour ma première course, j’ai neuf ans. On progresse et on commence à gagner des médailles. On est assez paresseux à lécole et le débarquement américain de novembre 1942, l'opération Torch, provoque du remue-ménage. 59

De même, la nageuse Héda Frost, née en 1936 à Alger, et dont le père issu d'une famille aisée d'hôteliers tchèques néchappe pas au déclassement social en devenant chef de rang, voit aussi sa scolarité écourtée à 14 ans au gré des déplacements familiaux.

Vendeuse au Petit Duc d'Alger, elle se souvient de ses débuts de nageuse, comme d'autres hors d'un cadre fédéral largement développé et où l'on recense en 1954 plus de 75000 licenciés dont un tiers de footballeurs ${ }^{60}$ :

Je fais partie de la première équipe de volley-ball de l'Association Sportive Municipale d'Alger. Et comme je nage naturellement en bord de mer, on m'incite à m'inscrire dans un club. Je rejoins le Groupe Laïque d'Études d'Alger, une organisation multisports visionnaire ouverte sur la jeunesse et les étudiants. Il y une piscine couverte de 25 mètres praticable toute l'année. Le nouvel entraîneur venu du nord de la France, le champion Émile Soron, me prend en main. Je le retrouve chaque matin à six heures et le soir, après mon difficile travail fini. Avec Marc Kamoun, nous sommes des purs produits du GLEA. Nous nous déplaçons régulièrement dans toute l'Afrique du Nord et parfois en métropole pour des compétitions que nous remportons. En 1955, juste avant ma première sélection en équipe nationale, la Fédération Française de Natation s’aperçoit que je ne suis pas française. Avec Monsieur Baroli, le président de la ligue d’Alger, nous allons à la préfecture. Arrivée à Paris, j’ai ma carte d'identité française. C’est la magie du sport. ${ }^{61}$

Pour ces pratiquants, les recrutements et la progression de leurs excellences sportives dans des clubs algériens où ils trouvent des entraîneurs vus comme des "seconds pères" charismatiques et des présidents notables sont cruciaux. Tous les novices sont repérés et pour les meilleurs, surclassés. Autrement dit, leurs 
carrières algériennes déterminent leurs futures performances de haut-niveau en France. Des dizaines de titres s'accumulent offrant à ces espoirs du sport algérien une notoriété locale puis nationale. En 1955, la presse algéroise est bien présente pour couvrir un événement populaire: Marc Kamoun remporte la Coupe de Noël, consistant en la traversée du port d'Alger, précédant son amie Héda Frost qui devance pour l'occasion tous les autres hommes engagés.

Outre le huppé RUA d'Albert Camus, parangon d'une Algérie semblable à une seconde France, ${ }^{62}$ et le GLEA où évolue l'athlète olympique Simone Brierre, trois clubs concurrents forment des futurs sélectionnés olympiques de natation: Bridja Sports à Alger (Jean-Pascal Curtillet et René Million), Glorieuse Marine Oranaise (Françoise Borie et Alain Gotvallès) et Union Nautique Indépendante Constantinoise (Maryse Morandini et Guy Montserret). D’ailleurs, le climat méditerranéen d'Algérie et les 1200 kilomètres de côte ne peuvent pas expliquer que 44\% des Français d’Algérie sélectionnés aux Jeux le soient dans des disciplines aquatiques comme l'aviron, la natation, le pentathlon, la voile et le water-polo. Le sport fait une percée, favorisé par l'urbanisation, l'explosion démographique, les progrès de la scolarisation et la présence de 400 installations sportives modernes: stades, terrains de sports et d'éducation physique auxquels s'ajoutent piscines et vélodromes. ${ }^{63}$ Environ cinq milliards de francs sont dépensés pour léquipement sportif des départements algériens de 1949 à 1959. ${ }^{64}$ Parfois, des rencontres improbables se produisent. Né en 1943 à Oran, Jean-Paul Anton fait la connaissance de Roger Tessier, l'un des gardes du corps du Général de Gaulle, alors entraîneur du boxeur d’origine espagnole José Hernandez qu'il mène deux fois au Championnat d'Europe:

J'ai 10 ans et demi, assez bagarreur. Un beau jour, un gars se présente au garage à domicile de mon père pour réparer sa BMW. À la demande de mon père, il m’accepte dans sa salle de boxe. Plus tard, je fais sous son autorité une dizaine de combats. Il part à Paris en 1958 quand de Gaulle revient au pouvoir. Je ne pense qu'à m’amuser, me battre et faire des conneries. Je suis embarqué avec l'OAS. ${ }^{65}$ On recrute dans les collèges et lycées. Je ne passe pas mon bac. Le jour du putsch d'Alger, le 23 avril 1961, mon père me met sur un bateau. Je quitte l'Algérie pour rejoindre Roger Tessier. Je débarque à Marseille puis monte à Paris. Je vis chez les Tessier pendant quelques mois. Ils deviennent comme mes parents adoptifs. Pendant toute ma carrière, Roger Tessier est mon seul entraîneur, ce qui déplait aux cadres de la fédération. ${ }^{66}$ 
Les accointances de Jean-Paul Anton avec l'OAS (distribution de tracts, manifestations bruyantes et réunions secrètes) partagées par le pentathlète Jean-Pierre Giudicelli comme les attentats commis par le Front de Libération Nationale ${ }^{67}$ sur les pères du gymnaste Henry Boerio et des pentathlètes Alain Cortès et Lucien Guiguet, rappellent la perméabilité des frontières entre sport et politique. Les circonstances particulières de la Guerre d'Algérie qui cause la mort de plusieurs centaines de milliers d'hommes bouleversent leurs destins.

\section{Les filières dans la guerre}

Les conditions de vie misérables des musulmans se durcissent par les privations de la Seconde Guerre mondiale, leur déscolarisation et la crise agraire qui aggrave les déséquilibres économiques. À la Toussaint rouge du $1^{\mathrm{er}}$ novembre 1954 succède la campagne de boycott de tabac et des alcools lancée par le FLN et surtout le terrible soulèvement nord-constantinois du 20 août 1955: 123 civils sont massacrés dont 71 dans la population européenne. La répression prend l'allure d'une guerre. Les effectifs militaires sélèvent à 190 ooo hommes en février $1956 .{ }^{68}$ De nombreux musulmans sont assassinés par l'armée française dans le stade de Philippeville. ${ }^{69}$ Quelques mois plus tard, le nombre de soldats a presque doublé, s'appuyant sur l'engagement des harkis. ${ }^{70}$ Les représailles aveugles cimentent l'unité nationale des musulmans contre les Français. Le point de non-retour de la révolution algérienne est atteint. ${ }^{71}$ Chaque jour, les sentiments d'injustice et de peur se font plus pressants. Au début du conflit armé, jusqu’en 1956, les métropolitains, peu informés, sont globalement indifférents à l'exception de quelques familles de jeunes appelés. La société française refuse de se vivre en état de guerre. Mais en Algérie, les tensions se renforcent. Au même moment, le FLN lance une série d'attentats. Le couvre-feu est imposé à Alger. Les "pouvoirs spéciaux" sont confiés à l'armée et suspendent ainsi la plupart des garanties de libertés individuelles. Trois zones (pacification, opération et interdite) séparent le territoire algérien, provoquant d'arbitraires déplacements de population. L'intensification de la Guerre d'Algérie qui véhicule d'autres enjeux dont la présence d'un colonat blanc de près d'un million de personnes et la mobilisation des appelés, au cours des années 1956-1957, oblige chacun en métropole à se positionner par rapport au conflit. Ce questionnement est tardif et brutal car un consensus existe autour de la question coloniale depuis l'entre-deux-guerres..$^{72}$ 
En 1956, le FLN ordonne aux clubs musulmans et aux footballeurs musulmans des clubs européens de cesser toute activité sportive impérativement. L'instruction est suivie par les Algériens sportifs et artistes qui désertent les stades et les scènes de théâtre. Les championnats de la ligue d'Afrique du Nord se trouvent perturbés et la machine sportive grippée. Pour incarner la dynamique de rupture avec la société coloniale, les premières bombes explosent simultanément dans les stades d’Alger à Belcourt et El Biar le 10 février 1957 faisant 11 morts et 45 blessés. Voie privilégiée de survie identitaire, d’auto-persuasion existentielle et de renforcement de la fraternité sportive avec la métropole, les équipes européennes gagnent tous les titres. Concrétisant un rêve d'intégration de plus en plus menacé, un match de football opposant la sélection d'Alger à l'Équipe de France réserve est organisé en 1958. En 1959, l’année du Plan de Constantine, propagande oblige, les clubs algériens sont autorisés à participer au Championnat de France Amateur en constituant un sixième groupe de 10 clubs jusqu'en mars $1962 . .^{73}$ Le sport a finalement peu d'impact dans cette contre-offensive coloniale visant à amarrer l'Algérie à la France. Les Foyers Sportifs des Sections Administratives Spécialisées chargés d’initier les Algériens aux sports individuels et collectifs n'y changent rien. Les violents affrontements communautaires et la folie sanguinaire submergent l'Algérie. Didier Rey rappelle que la situation s'aggrave durant la dernière année de la guerre. Bien avant les enlèvements et les massacres de plusieurs centaines d’européens à Oran au lendemain de l'Indépendance, les stades servent de nouveau d'antichambre de la mort, comme le 5 juillet 1962, où de nombreux Européens sont emmenés au stade municipal d'Oran et assassinés pour la plupart.74

Quand ils quitteront le joyau de l'empire, les Européens auront largement contribué au développement du sport local. Dans leur paradis perdu, ils laisseront des infrastructures uniques en Afrique comme le Centre Régional d'Éducation Physique et Sportive flambant neuf de Ben Aknoun, des milliers de sportifs formés par leurs soins et des vedettes de toutes religions. Dernier épisode de la tragédie algérienne, l'exode de près d'un million de pieds-noirs se dessine. Choisissant la valise au cercueil, ils abandonnent du jour au lendemain leur terre natale, leurs biens et leurs morts dans la pagaille indescriptible d'une des plus grandes migrations du XX ${ }^{\text {ème }}$ siècle.75 C’est l'arrachement vertigineux, une fuite inévitable et incompréhensible. L'historien Yann Scioldo-Zürcher précise que:

Une fois reconnu comme tel, chaque rapatrié peut bénéficier de prestations de retour, de subsistance, de prestations sociales et d'une aide au reclassement. 
[... Chaque ménage rapatrié perçoit, pendant une année, dans l’attente de retrouver un emploi, une allocation dite de subsistance. [ . . . ] Si la loi du 26 décembre 1961 prévoit l'indemnisation des biens abandonnés outre-mer, sans préciser le montant de sa mise en place, elle privilégie surtout l'intégration des rapatriés par le travail. ${ }^{76}$

Et pourtant, le départ est irréversible.

Bien avant l'embarquement de Jean-Paul Anton, l'International Joseph Bernardo, contacté par Alfred Nakache, s'installe à Toulouse en 1951 et évolue au Toulouse Olympique Employés Club du célèbre Alban Minville, attentif à ce juteux réservoir algérien. Évitant aussi le défi existentiel de l'exode, Marc Kamoun, né en 1937 à Alger, rejoint la métropole en 1956 en tant quétudiant et sociétaire du Racing Club de France de Pierre Barbit:

Je viens d’obtenir mon second bac à Alger. Ça commence à barder avec la Guerre d'Algérie. Le FLN s'attaque aux étudiants et aux jeunes pour marquer le coup. Il pose des bombes dans les cafés, les facultés et les boites de nuit. Un jour, en sortant de la fac, je dois me trouver dans un café plastiqué. Le lendemain, mon père très inquiet m’expédie à Paris pour que je m’inscrive en Pharmacie. Je ne connais personne à l'exception d'une relation de mon père, un pharmacien, qui accepte de me prendre comme stagiaire et qui me trouve une chambre chez l'habitant. Je me tourne vers les nageurs que j’ai rencontrés aux championnats de France. En tant que sprinter, je sais que le meilleur club de Paris est le Racing. J'y signe une licence sans problème. Ma véritable carrière commence..$^{77}$

Elle dure jusqu'en 1962. Cette année-là, vêtue d'une tenue blanche recouverte de pieds-noirs, Héda Frost monte fièrement sur la plus haute marche de son dernier podium aux Championnats de France. Plus tard, elle regrettera les réticences du mouvement sportif et l'angoisse des métropolitains dêtre détrônés:

On vit au milieu d'une insécurité totale. Je pars avant la débâcle. En mai 1962, à la demande de Monsieur Baroli, jemmène avec moi en avion à Paris un groupe de jeunes nageurs pour une compétition entre le Nord et le Sud. Le Colonel Crespin ${ }^{78}$ me demande de rester pour les préserver. Nous sommes logés à l'Institut National des Sports (INS). Je reprends l'entraînement. En septembre, je retrouve mon mari, encore directeur de l’antenne d’Alger de la Compagnie Générale d'Électricité. C’est 
l'éclatement de l'Algérie. Je reviens en France en 1963. Au niveau de l'accueil, rien nest fait pour nous. Seul le Colonel Crespin nous aide quand on lui demande. Les autres restent indifférents. ${ }^{79}$

Quant à Jean-Pierre Giudicelli, il devance l'appel sous les drapeaux et s'engage dès 1961 au premier régiment de Parachutistes d'infanterie de Marine de Bayonne. Il y est rejoint deux ans après par Lucien Guiguet, avec qu'il tisse une durable amitié. Ce dernier, né à Cherchell en 1942, relate son été 1962 à Constantine, la ville des " ponts suspendus » où il s'exerce près des gorges du Rummel dans tant de rivières et de bassins naturels:

Mon père est militaire, maitre armurier. Son père fait partie des premiers piedsnoirs d'Algérie. Mon père fait sa carrière en France et en Allemagne. En 1959, il est remuté en Algérie. Il pense y prendre sa retraite et reprendre la ferme de son frère décédé et son café-restaurant. Nous vivons à Constantine dans le quartier arabe de Sidi Mabrouk supérieur. Nous ne vivons pas en caserne. Je suis Scout de France. Lycéen, je passe mon brevet de maître-nageur à 18 ans dans un club. Mon père prend une balle en descendant de sa jeep. Le rapatriement en septembre 1962 se passe plutôt bien. Le seul problème, c'est qu'il faut trouver un déménageur. Soutenu par ladministration militaire, nous débarquons à Marseille et nous rendons dans notre Citroën Traction à Offenburg où mon père est nouvellement affecté. ${ }^{80}$

Cette filière militaire fait écho aux réseaux sportifs en pleine consolidation. La France est déjà une terre d'immigration sportive attractive.$^{81}$ Née en 1947 à Oran, la nageuse Françoise Borie, fille d'un aisé restaurateur propriétaire du Café de Clichy rue d'Arzew, saisit sa chance. Elle quitte un pays en feu:

En Algérie, on est français avant tout. On forme une communauté. En 1962, tout le monde pense quavec la base navale de Mers-el-Kébir, Oran restera français. J'ai 15 ans. Je vais rentrer en première. J'entretiens une correspondance avec des nageurs des Dauphins du TOEC car je suis déjà en Équipe de France junior. Ils maccueillent et me logent chez une nageuse à Toulouse. Mes parents restent en Algérie. Je débarque le 13 juin à Port-Vendres avec une sœur de ma grand-mère et quelques valises. Rien n'est fait pour nous. On retrouve à Toulouse ma grande sœur et nous installons dans un studio puis un appartement. ${ }^{82}$ 
Résidant souvent dans le Midi où se trouve la moitié des réfugiés pour se rapprocher le plus près possible de l'Algérie interdite, ils savent que tout est à refaire.

À l'image d'une nouvelle génération qui éclot, de nouvelles figures sportives d'Algérie apparaissent. Contrairement à leurs aînés voyant le jour dans les années 1930 et sélectionnés olympiques avant l'Indépendance, ces jeunes découvrent le sport fédéral en France où ils grandissent. Le 4 juillet 1962, depuis trois jours à la base aérienne La Senia d'Oran, sous protection militaire, Alain Cortés, âgé de 10 ans, monte avec ses parents dans une Caravelle qui l'éloigne du danger. Son père policier est employé comme typographe dans une imprimerie d'Uzès puis une usine de Bagnols-sur-Cèze avant deetre réintégré quelques années plus tard. En 1961, à moins d'un an, l'algérois William Ayache, futur champion olympique de football, ne peut pas ressentir les sentiments d'incertitude de ses parents et de ses nombreux frères et sœurs. Ils laissent derrière eux leur grande boucherie casher:

Même si on a de la famille à Marseille et Paris, on atterrit dans le Sud-Ouest, à Tarbes, avec une valise, parce que ma mère trouve un emploi dans une clinique. Mes parents perdent tout. Donc, il faut se reconstruire et s'intégrer. On s'installe dans la cité HLM Bel-Air au milieu d'autres communautés assez unies entre elles: des Portugais, des pieds-noirs, des arabes, des Espagnols. Il y a quelques années difficiles mais à force d'humilité et de respect, mes parents savent se faire apprécier. ${ }^{83}$

Considérés comme des "arabes" aux yeux de l'amalgame, ils ne prêtent bientôt plus attention aux insultes de la cour de récréation. C'est dans un autre contexte qu'Henry Boerio et sa famille quittent Saint-Arnaud et s'installent à Montceau-lesMines en 1959. Rendu paraplégique par un attentat survenu dix-huit mois plus tôt, son père à la tête d'une exploitation agricole n'est plus en mesure de travailler. Il décide d'utiliser ses relations amicales en Saône-et-Loire issues de son engagement dans le troisième régiment de parachutistes de la France Libre, les Special Air Service. Les anciens maquisards et médecins militaires restés solidaires s'engagent à l'aider à s'installer en métropole. Pour le clan Boerio, la vie redémarre dans une maison meublée prêtée de la cité ouvrière et des soins quotidiennement prodigués. Résilients, tous ces jeunes sportifs trouveront dans leur club un espace de solidarité et de réassurance. ${ }^{84}$ 


\section{Le perfectionnement sportif en France}

Laccession à l'indépendance des colonies françaises d'Afrique, la fin de la Guerre d'Algérie, le renforcement de la Guerre Froide, les grèves des mineurs des Houillères en mai 1968, la féminisation du secteur tertiaire et la mixité dans les nouveaux établissements scolaires sont quelques indicateurs des ruptures politiques, économiques et culturelles de la France des années 1960. Sur le plan sportif, le développement du sport fédéral, l'apparition de pratiques libres de loisirs sportifs, le processus de sportivisation de léducation physique font écho à l'avènement du sport télévisuel. ${ }^{85}$ D'après les données recueillies, à l'exception d'Héda Frost qui mène sa carrière de nageuse exclusivement au GLEA, les clubs français spécialisent ces sportifs grâce à des entraînements plus nombreux. Seule la discipline les fait progresser. Individuellement ou collectivement, leurs performances augmentent. C'est en 1951, dans la piscine découverte du Cercle des Nageurs de Marseille auquel s'affilieront Monique Pietri, Pierre Amardeilh et Pierre Garsau que Joseph Bernardo, déjà proche du Cercle, bat le record du monde du relais quatre fois 200 mètres nage libre. La camaraderie et la rivalité avec Jean Boiteux, Willy Blioch et Alex Jany, le stimulent. Cependant, il insiste sur le rôle décisif joué dans son parcours par sa signature au TOEC: "Le club toulousain me reçoit très bien. Il y a une grosse équipe avec Alex Jany, Alfred Nakache, Georges Vallerey et Charles Babey. On est des artisans et on aime cela à la folie. Il faut être sérieux. Je ne travaille pas à côté. Il ne faut pas parler d'argent sinon on est disqualifié à vie. Le club nous en donne un peu, mes parents aussi. Cela me permet de vivre à l'hôtel et de manger tous les jours." ${ }^{86}$ Quatorze ans plus tard, en 1965, Jean-Paul Anton de l'Association Sportive Simca Poissy est Champion de France poids plumes. Alors que s'affirme l'élan modernisateur de la politique sportive gaullienne de soutien à la haute-compétition, il peut aussi compter sur les autres boxeurs amateurs et professionnels de la salle du manager Philippe Filippi. ${ }^{87}$

Avec l'adoption en juin 1973 de la Charte du football professionnel, la formation se rationnalise et les premiers centres de formation sont créés. Larrièregauche William Ayache quitte en 1977 Tarbes PF pour poursuivre avec succès son apprentissage au FC Nantes dans le centre sportif de la Jonelière inauguré en 1978. L'entrée en formation constitue à la fois une promotion sportive élective, marquée par un rapprochement avec l'espace strictement professionnel du football, et une rupture plus ou moins nette avec le cadre de la pratique et la façon 
de jouer antérieurs. Le recrutement au centre participe à la construction de la vocation, la croyance "dêtre fait pour ça." ${ }^{88}$ Dès 1979, coupé du milieu familial, il dispute son premier match en première division et rejoint l'équipe nationale en 1983. Un an plus tard, emmené par Henri Michel, avec ses équipiers Didier Sénac et Guy Lacombe, il décroche l'or olympique à Los Angeles. Il travaille dans huit clubs professionnels en France; une mobilité notable contrastant avec l'inscription durable dans un club des sportifs amateurs questionnés. Mieux défendu et mieux payé, il n'est pas non plus épargné par des blessures qui nuisent à son évolution. ${ }^{89}$ Comme d'autres, Henry Boerio, né en 1952 à Sétif et devenu Pupille de la Nation, se remémore la rigueur, l'assiduité et l'ascétisme dont il doit faire preuve, soutenu par ses proches:

Je signe ma première licence en octobre 1960. Dans les six premiers mois, je gagne mon premier concours au sein du club. Une victoire en appelle une autre. Il n'y a pas encore de section sport-études. Je vais au lycée et m’entraîne chaque soir. Je suis alors membre de l'Équipe de France junior. Mon entraîneur est Joseph Sniezek. Je passe le bac et veux préparer le professorat d'éducation physique et sportive à l'Université de Dijon. Pour m’entraîner avec les jeunes talents du centre national d'entraînement de Montceau-les-Mines ouvert en 1972, je fais 110 kilomètres aller et 110 retour. Je sors des cours à Dijon plus tôt que prévu. Je prends le train. Mon père dans son véhicule aménagé me récupère à la gare. Il m’emmène au gymnase. À la fin de l’entraînement, maman arrive avec un petit panier repas. Elle me ramène à la gare. Je mange dans le train retour. Mon frère mattend à Dijon et nous allons dormir à la cité universitaire. Pendant ma carrière, il y a au minimum six heures d'entraînement par jour du lundi au dimanche. Tout est réglé par l'entraînement et la compétition..$^{\circ}$

À l'instar d'autres recherches restituant les déterminants sociaux des performances de haut-niveau, l'armée française les influence..$^{11}$

Certains comme Marc Kamoun et Alain Cortès intègrent le prestigieux Bataillon de Joinville à la Faisanderie dans l'est parisien comme "athlètes qualifiés." Dans cette école du sport de haut-niveau, ils s'entraînent dans des conditions dignes des professionnels tout en recevant une instruction militaire. L'armée des champions n'est pas la seule à les favoriser. ${ }^{92} \mathrm{Au}$ premier régiment de train de Montlhéry en 1961, Jean-Paul Anton bénéficie d’un régime dérogatoire favorisant ses progrès et l'obtention d'un titre de champion de France militaire. 
Cette première récompense a une saveur particulière et provoque la fierté familiale. Pour la première fois, la France le reconnaît. Avec l'appui de l'influent Roger Tessier, sa conscription se fait proche de Paris et loin de l'Algérie. Les permissions de sortie sont nombreuses. En 1966, grâce à leurs performances en pentathlon militaire, les jeunes parachutistes de Bayonne Jean-Pierre Giudicelli et Lucien Guiguet sont spécialement recrutés à la caserne Nansouty de Bordeaux par le Colonel Émile Gueguen avec pour seule mission de préparer les Jeux de Mexico en 1968. Le "sport à outrance"-pour reprendre l'expression de Jean-Pierre Giudicelli93 - est ordinaire: du matin au soir, sans relâche, les cinq exigeantes disciplines du pentathlon moderne (escrime, natation, équitation, tir au pistolet et course à pied) sont travaillées jusquà l'épuisement. Ces sportifs amateurs encore peu médiatisés, voyageant en Europe, aux États-Unis et parfois en Chine, ${ }^{94}$ ne perçoivent pas leur sport de prédilection comme une activité offrant un avenir stable. Pendant leur carrière, selon leurs parcours et leurs diplômes, ils sont étudiants, professeurs d'éducation physique et sportive, maître d'armes ou encore militaire de carrière. Tous jouissent d'horaires aménagés qui ne sont pas autant confortables que le statut de footballeur professionnel de William Ayache dont la longue carrière, forte de 400 matchs, s'achève en 1995. En sacrifiant leur temps libre à l'entraînement et à la compétition, en s'imposant un nouveau rythme de vie (nutrition, récupération et sommeil) marqué par la recherche du dépassement de soi et l'exténuation, rares sont les célibataires qui établissent pendant leurs carrières de nouvelles relations amoureuses durables.

Des décennies après leur consécration olympique, les 10 sportifs interviewés expliquent que le succès grandissant des Jeux tient à l'augmentation de la qualité de l'organisation, la reconnaissance du public et l'intérêt des médias. Ils soulignent également l'accroissement du niveau de performances lié aux sauts technologiques du matériel, la préparation optimisée des sportifs, la spécialisation, la concurrence internationale et l'irruption d’enjeux politiques et économiques. Ils y participent en moyenne 1,6 fois entre 1948 et 1984, obtenant une médaille d'or (William Ayache en 1984) et plusieurs de bronze (Joseph Bernardo en 1948 et 1952, Jean-Pierre Giudicelli et Lucien Guiguet en 1968 et Henry Boerio en 1976). L’un des plus grands nageurs de l'après-guerre, "Jo," ranime la flamme de ses premiers Jeux durant lesquels il croise les athlètes Micheline Ostermeyer et Alain Mimoun: "Londres, c'est les Jeux de la paix. Il y a une certaine euphorie, même si c'est le temps de la reconstruction et qu'il y a beaucoup de restrictions. À la cérémonie d’ouverture, on est canardé par des pigeons. Heureusement qu’on 
porte le béret basque!"95 Les sportifs sont alors hébergés dans des baraquements de l'armée. Pour ses podiums à Londres et Helsinki, ils sont est reçus deux fois à l'Elysée par le Président Vincent Auriol. En 1960, à Rome, Marc Kamoun retrouve Héda Frost qui garde de "magnifiques souvenirs des Jeux de Melbourne de 1956, une aventure dans un pays lointain, une ambiance de fête entre sportifs sérieux." 96 Il vit son rêve olympique:

Tout en suivant mes études de kiné, je veux absolument gagner ma place pour les Jeux qui ont plus d'aura que les Championnats d'Europe. C'est le but de ma carrière. J'ai 23 ans. Je me qualifie pour le relais quatre fois 200 mètres nage libre. Léquipe est constituée de Jean Boiteux, Gérard Gropaiz et Jean-Pascal Curtillet, mon copain du Racing. On échoue. L’expérience olympique reste inoubliable. C'est très décontracté. Il n'y a pas de service d’ordre ni de professionnels. Dans le village olympique, tout le monde se côtoie. ${ }^{97}$

Mais l'émerveillement peut laisser place à la suspicion.

Tous sont sensibles à la diffusion du dopage, faussant les hiérarchies. Lucien Guiguet, le médaillé de Mexico, parle encore avec émotion de l'été 1968 où sont brandis les poings gantés des athlètes afro-américains Tommie Smith et John Carlos:

On ne va pas aux Jeux pour la gloire ni pour l'argent. On gagne l'équivalent de 15 francs par jour. Nous terminons quatrièmes de lépreuve derrière les Suédois mais ils sont disqualifiés pour dopage. À lépoque, on se dope à l'alcool. Tout le monde le fait. L'alcool est un moyen efficace pour se désinhiber, notamment pour le tir ou l'escrime. À Mexico, j’apporte mes bouteilles de Kronenbourg Special pour être sûr de ne pas dépasser le seuil règlementaire de 0,4 grammes. Le problème des Suédois est qu'ils forcent la dose. Finalement, avec Jean-Pierre Giudicelli, Raoul Gueguen et Christian Châtillon, nous sommes reclassés troisièmes et terminons notre aventure mexicaine par un détour à Acapulco entre jeunes. ${ }^{98}$

Sous couvert du recrutement d'entraîneurs étrangers, la préparation physique se "médicalise." Naïf ou bien conscient des risques encourus à l'époque, Jean-Pierre Giudicelli confie courageusement qu'au début des années 1970, "le nouveau directeur technique hongrois de l'Équipe de France, un certain Lazlo, nous incite à manger des 'œufs' pour 'préparer le cœur.' Il faut les prendre avec du lait. Je 
rentre dans le jeu. J'essaye mais j'arrête très vite."99 Il n'est certainement pas le seul dans cette situation embarrassante qui pousse certains, lassés et arrivés à saturation, à mettre un terme à leur carrière de haut-niveau.

Après une dernière olympiade, ils prennent leur retraite sportive en moyenne à 28 ans et deux mois. Revenu à la vie civile, le natif de Blida Alain Cortès qui bénéficie jusque-là d'une bourse dérisoire "Promotion Spéciale" de l'INS et de primes de préparation olympique, fait un choix évident en 1982: "Depuis des années, je souffre du dos. On me diagnostique une spondylarthrite-ankylosante. J'ai des poussées inflammatoires répétitives, même en compétition. Je dois arrêter ma carrière à 30 ans. Ce qui me fait quitter le pentathlon, c'est aussi le dopage que je ne veux pas cautionner." ${ }^{100}$ Si l'exercice d'un métier, le cumul des capitaux matériels et symboliques et les compétences acquises peuvent assurément être une ressource pour la reconversion, rien ne semble préparer les sportifs au "choc biographique"101 que constitue la fin de carrière. C’est une "épreuve de la petitesse." ${ }^{102}$ Ils redoutent l'isolement que subit l'oranais Alain Gotvallès, recordman du monde du 100 mètres nage libre en 1964, qui, exclu de son univers de prédilection, décède prématurément à 66 ans comme agent d’assurances.

\section{Les bénéfices du capital sportif}

Au-delà des politiques ministérielles et fédérales, les conditions de sortie du dispositif de performance sont le produit d'une trajectoire à penser dans son ensemble. C'est ce que tend à prouver en 2011 l'enquête des sociologues Sébastien Fleuriel et Manuel Schotté sur le devenir des sélectionnés français aux Jeux de Munich en 1972 et de Barcelone en 1992. ${ }^{103}$ Ils avancent que si les enquêtés accèdent à des positions majoritairement situées dans le haut de l'espace social, celles-ci restent la plupart du temps ancrées dans l'espace sportif, produisant une sorte d'enfermement. Ces deux chercheurs évoquent une tension paradoxale entre les situations sociales observées chez les anciens sportifs qui traduisent pas ou très rarement de déclassement objectif et la perception négative que certains d'entre eux se font parfois de cette même situation. Une quinzaine d'années plus tôt, Pierre Lanfranchi et Alfred Wahl s'intéressent au devenir des anciens footballeurs professionnels des années 1930, leurs tentatives de reconversion comme entraîneur, les achats de bar-tabacs dans les années 1950, l'investissement dans les magasins de distributions d'articles de sport dans les années 1960 et 1970 
et bien sûr la difficile acceptation de l'anonymat. Si la carrière de footballeur professionnel est rarement à l’origine d'une ascension sociale durable, pour des centaines de joueurs, elle est le marchepied pour quitter la condition ouvrière..$^{104}$ Malgré de récentes recherches, ${ }^{105}$ les repères historiques manquent au sujet de la reconversion des anciens sélectionnés olympiques.

Hormis Joseph Bernardo qui, grâce à son mariage avec la comédienne et metteuse en scène Simone Turck devient en 1954 régisseur du Théâtre du Grenier de Toulouse puis du Théâtre du Gymnase de Marseille, ${ }^{106}$ les positions des anciens sportifs restent systématiquement dans le champ socio-sportif. À la faveur dopportunités et de formations certifiantes, ils deviennent dans toute la France et aux Antilles conseiller technique régional (Jean-Paul Anton), entraîneur national (Alain Cortès), professeur d'EPS (Jean-Pierre Giudicelli), moniteur de sports militaire (Lucien Guiguet) et kinésithérapeute (Marc Kamoun). Comme ses anciens équipiers, William Ayache peine à s'imposer durablement dans le football professionnel. Depuis 1995, il est entraîneur adjoint de l'AS Cannes et du FC Mougins, consultant média et recruteur au FC Nantes. Laugmentation des charges d’entrainement et la multiplication des compétitions contraignent le sportif dans la construction de son avenir professionnel. Si le capital sportif constitue au sortir de la carrière une ressource déterminante pour l'accès à lemploi, il perd progressivement de son importance et sefface, que la reconversion des joueurs s'inscrive ou non dans l'univers sportif. Étudiante pendant sa carrière, Françoise Borie est diplômée puis rapidement nommée professeure de PhysiqueChimie au Lycée Climatique et Sportif de Font-Romeu.

Héda Frost retrace son parcours de jeune retraitée du sport dans la France des années 1960 et 1970:

Juste rentrée d'Alger, on me trouve en 1963 un poste de conseiller technique à la Jeunesse et aux Sports à Clermont-Ferrand. La Fédération de natation m’emploie pour organiser des stages de jeunes. Je viens ensuite à Paris avec un poste d'entraîneur nationale adjointe pour moccuper des jeunes à l'INS. Je rencontre évidemment Lucien Zins, le directeur technique national, une personne très ouverte. La Fédération m’envoie en stage aux Etats-Unis où je découvre de nouvelles méthodes d'entraînement. Je transmets ce que j’apprends aux jeunes, et notamment aux filles puisquà lépoque, elle s'entraînent à part des garçons. Je deviens directrice technique nationale de la natation féminine. Je reste en poste de 1964 à 1972. Après les Jeux de Munich, je moccupe pendant cinq ans à Vittel du Club 
Méditerranée qui subventionne une section sport-études natation. Ensuite, je m’installe à Aix-en-Provence où je prends en main un club. Je reviens aussi à Aix pour me rapatrier sur le Sud et m’occuper de mon fils. J’y prends ma retraite.107

Une nouvelle fois, différentes formes de patronages et de recommandation sont légion. Questions de génération, d'itinéraire et d’engagement intense dans le milieu sportif, elle est la seule sélectionnée olympique questionnée investie dans le Cercle Algérianiste. Née en 1973, cette association nationale cherche à "redonner ses lettres de noblesse à une culture pieds-noirs jusque-là marginalisée." ${ }^{108} \mathrm{Il}$ est d'ailleurs frappant de constater que ces sportifs pieds-noirs se connaissent peu. Grâce à ses engagements politiques précoces auprès du maire de Monceau-lesMines André Jarrot, Henry Boerio est contacté en 1984 à Los Angeles par Jacques Chirac qui le nomme conseiller de son cabinet à la Mairie de Paris. Lancien gymnaste de La Montcellienne couvre deux secteurs, le sport et les rapatriés d’Afrique du Nord, puis est élu conseiller régional d'Ile-de-France en 1992 avec le soutien d'associations pieds-noires telle que Le Recours, ${ }^{109}$ créé par les militants Guy Forzi et Jacques Roseau. Quatre ans plus tard, il est nommé directeur de l'Institut National du Sport et de l'Éducation Physique-où il a enseigné, devient en 2000 attaché olympique et termine sa carrière en 2018 comme inspecteur général du Ministère de la Jeunesse et des Sports.

Avec Françoise Borie, affectée au Lycée Français d'Oran de 1983 à 1989, "contente de faire découvrir [s] es racines et [s] on histoire à [s] es trois enfants," Henry Boerio est le seul sportif interrogé à séjourner en Algérie. À loccasion des Jeux Méditerranéens d'Alger en 1975, il traverse le miroir: "Même si je ne suis pas allé à Sétif, je reviens sur ma terre natale. C’est un moment fort. Je gagne. Je monte sur le podium. J'entends La Marseillaise. Je suis pris par la délégation. Après ma victoire, on se promène dans la ville, la Casbah et la pêcherie. Mon père me l'a décrite dans les moindres détails." ${ }^{111}$ Pour tous les autres, surtout ceux qui y ont grandi, la plaie est refermée mais la cicatrice demeure douloureuse. L’Algérie recouvre sans doute une réalité qui ne saurait remplacer l'éden évanoui, comme le précise Joëlle Hureau: "ce pays neuf, qu’on lui soit hostile, indifférent ou qu’on lui manifeste une sympathie lointaine, devient, malgré la géographie, une contrée différente. [... L L'Algérie nouvelle tend à devenir synonyme d'échec et de gabegie." "112 Inhabitable, elle reste un "pays dévasté" de la mémoire. ${ }^{1{ }^{113}}$ Ces champions manifestent enfin des sentiments ambivalents vis-à-vis de la France qui a pu les mépriser à leur arrivée. Le fait d’avoir porté ses couleurs aide peut-être 
ces patriotes à dissiper "l'image d'une France marâtre, celle qui les 'brade' et qui ne sait les accueillir." 114 Plus qu'une revanche, leur réussite dans le sport de hautniveau leur permet d'affirmer leur identité et leur offre protection et reconnaissance de l'État. ${ }^{115}$ Ils sont l'un des visages en France d'une communauté qui, une fois les premières difficultés économiques de l'installation surmontée, fait déjà l'objet en vertu de sa citoyenneté française d'une politique d'intégration inédite menée depuis le début des années 1960. Contrairement aux autres immigrés qui souffrent de la dégradation du marché du travail, ses membres accèdent de manière privilégiée à la fonction publique et obtiennent plus facilement leurs droits à la retraite. ${ }^{.16}$

\section{Conclusion}

Arrivés sur une terre hostile en 1962, les Français d'Algérie ont eu le sentiment que leur existence était contestée et que leur histoire était niée. Cette recherche focalisée sur les carrières, trajectoires et reconversions des sélectionnés olympiques pieds-noirs tente de construire un pont sportif entre des mémoires souvent opposées. ${ }^{117}$ Elles font écho aux fractures sociales et politiques contemporaines d'une société postcoloniale mieux saisie sous l'angle du sport. Cette contribution nourrit le dialogue entre deux communautés qui ont vécu sur le même sol et que l'histoire a séparées. Elle vise à combler un vide historiographique et révèle les parcours des sportifs délite méconnus en dépassant l'alternative amateursprofessionnels ou un cadre d'analyse strictement genré. ${ }^{18}$ Les réseaux de sociabilité de ces champions s'inscrivent dans des parcours migratoires individuels et des histoires collectives qui questionnent l'intégration aux valeurs républicaines. Dociles et peu concernés par les manifestations étudiantes et les grèves générales de Mai 1968, ces jeunes supportent peut-être plus que leurs homologues métropolitains les charges sportives pour faire rayonner la patrie à travers leur pratique intensive. Malgré l'inégale et aléatoire distribution des destins, plusieurs déterminants structurent leurs itinéraires. Leur jeunesse en ville dans des clubs algériens et français, les filières sportives dans la Guerre d’Algérie, le rôle de l'armée française, leur carrière majoritairement sous le statut amateur, leur consécration olympique et leur reconversion facilitée sont mis en évidence. Il semble que le capital sportif, sous influence d'autres capitaux (sociaux, scolaires, économiques et culturels), incarne un potentiel rempart contre le chômage, une 
ressource utile pour être employable malgré leurs vulnérabilités. En 1996, après les Jeux d'Atlanta, avec la retraite sportive du sabreur Franck Ducheix, né en 1962 à Renan, l'histoire des Français d'Algérie sélectionnés olympiques se referme, laissant derrière elle un remarquable héritage au sport français.

L'histoire est une discipline cumulative, par essence inachevée. Le recueil et l'interprétation de nouveaux témoignages et de sources manuscrites comme la presse et les archives des clubs et de l'armée seront des étapes précieuses pour approfondir la compréhension de ces brûlantes migrations sportives francoalgériennes. Les Archives Nationales d'Outre-Mer et le Centre de Documentation Historique sur l'Algérie d'Aix-en-Provence ainsi que le Centre de Documentation des Français d'Algérie de Perpignan pourront être sollicités. De fécondes perspectives de recherche se dessinent: la comparaison des élites sportives, médiatiques, culturelles et artistiques, leurs significations du point de vue communautaire; les conditions d'arrestation d'Alfred Nakache à Toulouse en 1943 dans lesquelles serait impliqué son rival devenu milicien Jacques Cartonnet; ${ }^{119}$ le rôle éventuel de la religion dans l'intégration en métropole sans oublier les liens entre le mouvement sportif colonial et l'embrigadement politique au sein de l'OAS. Dans la complexité de la réalité historique, deux autres thématiques s'avèrent particulièrement enthousiasmantes tant sur les possibilités d'affinement théorique que des recherches empiriques. La première porte précisément sur l'engagement des sportifs musulmans dans l'Équipe de France olympique comme le champion du marathon d'Amsterdam Boughera El Ouafi en 1928, décolleteur aux usines Renault de Billancourt. Centrée sur le football colonial algérien, la seconde s'intéresse aux 29 footballeurs européens portant le maillot tricolore de 1924 à $1959 .{ }^{120}$ Leurs noms évoquent aussi de belles pages du sport français. 


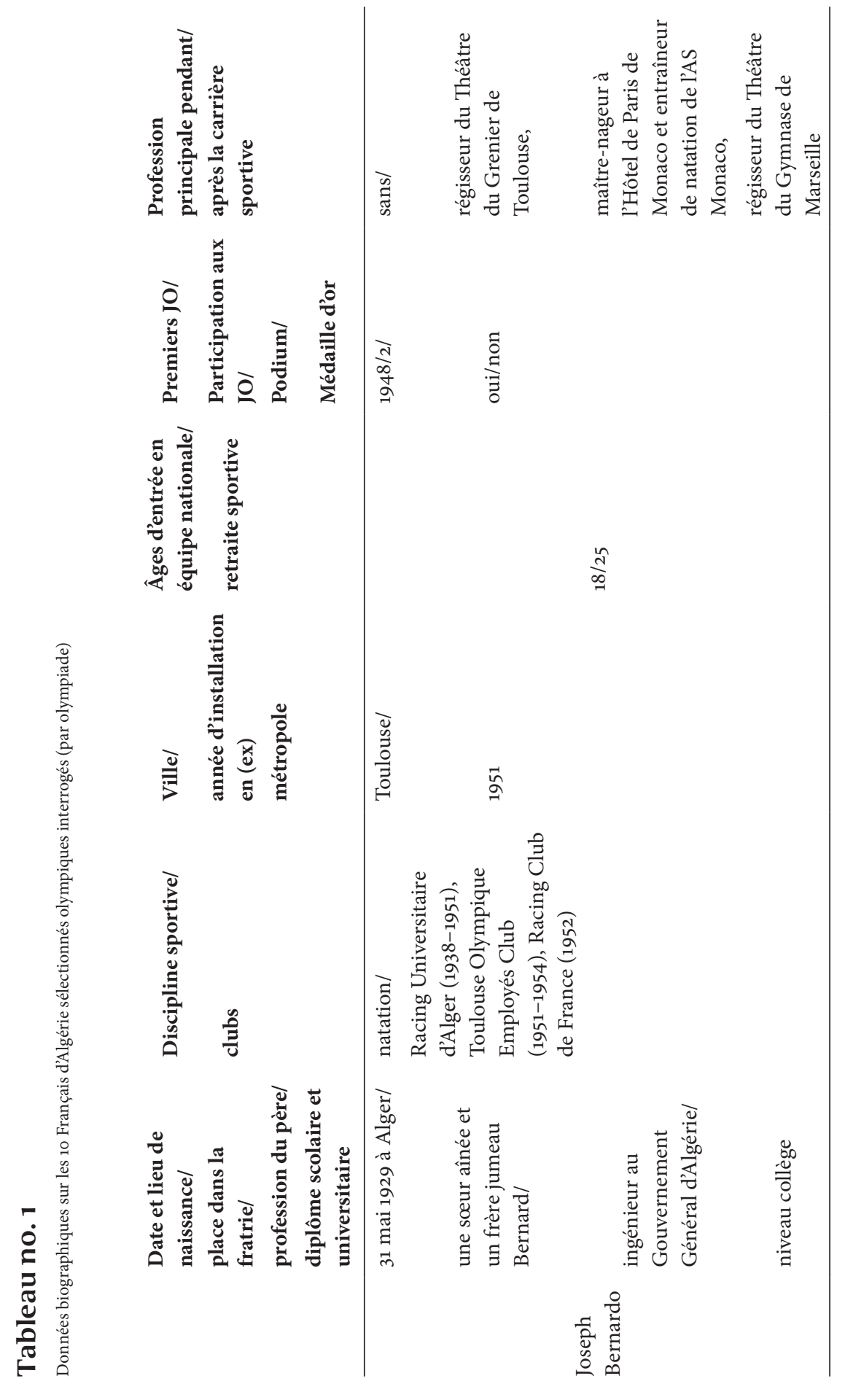




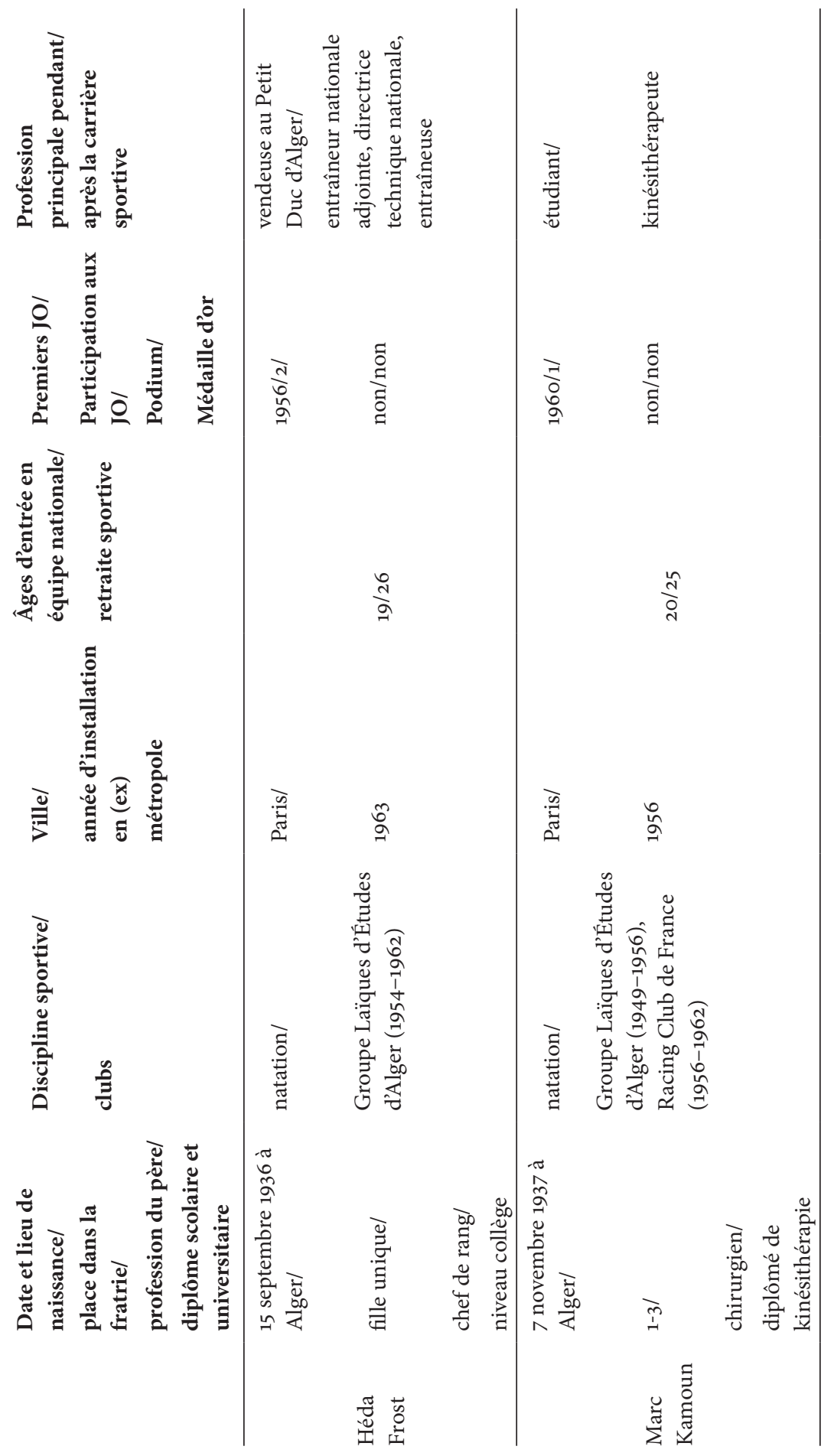




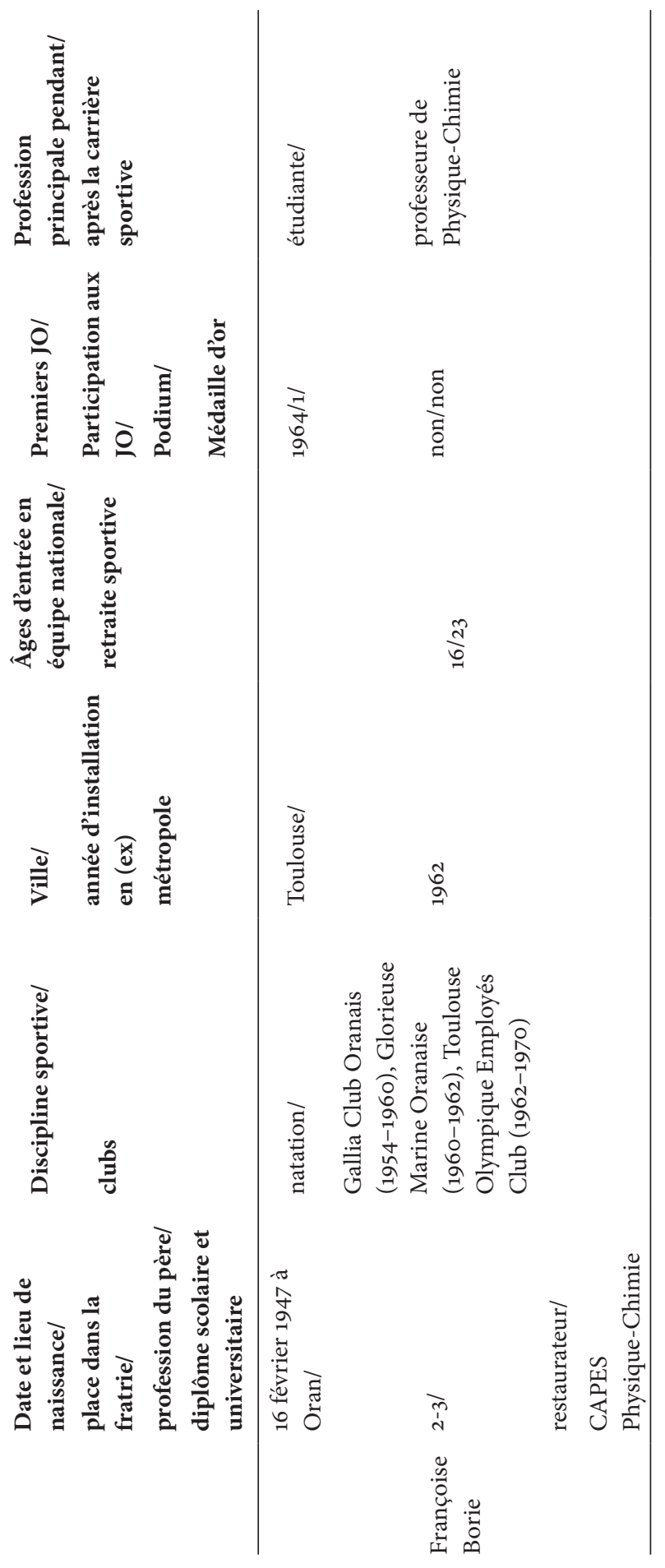




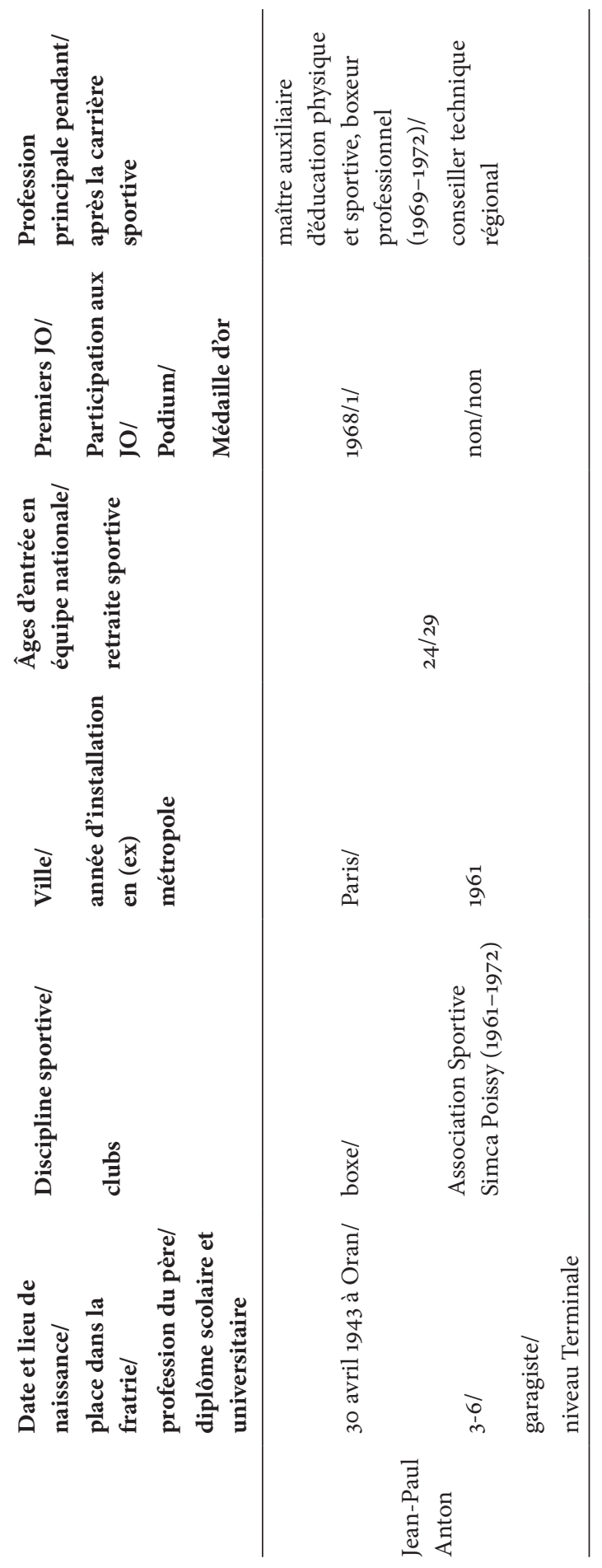




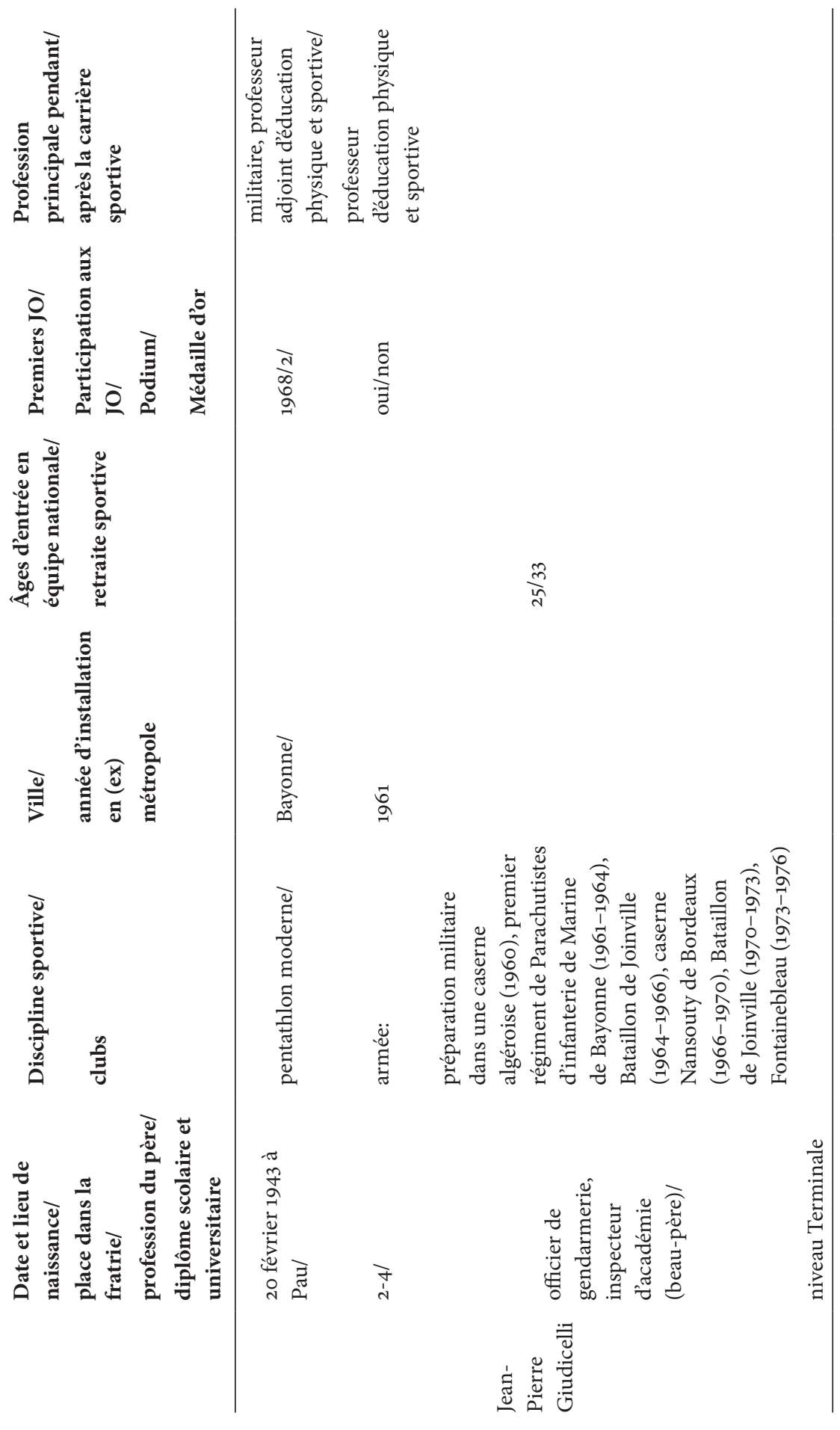




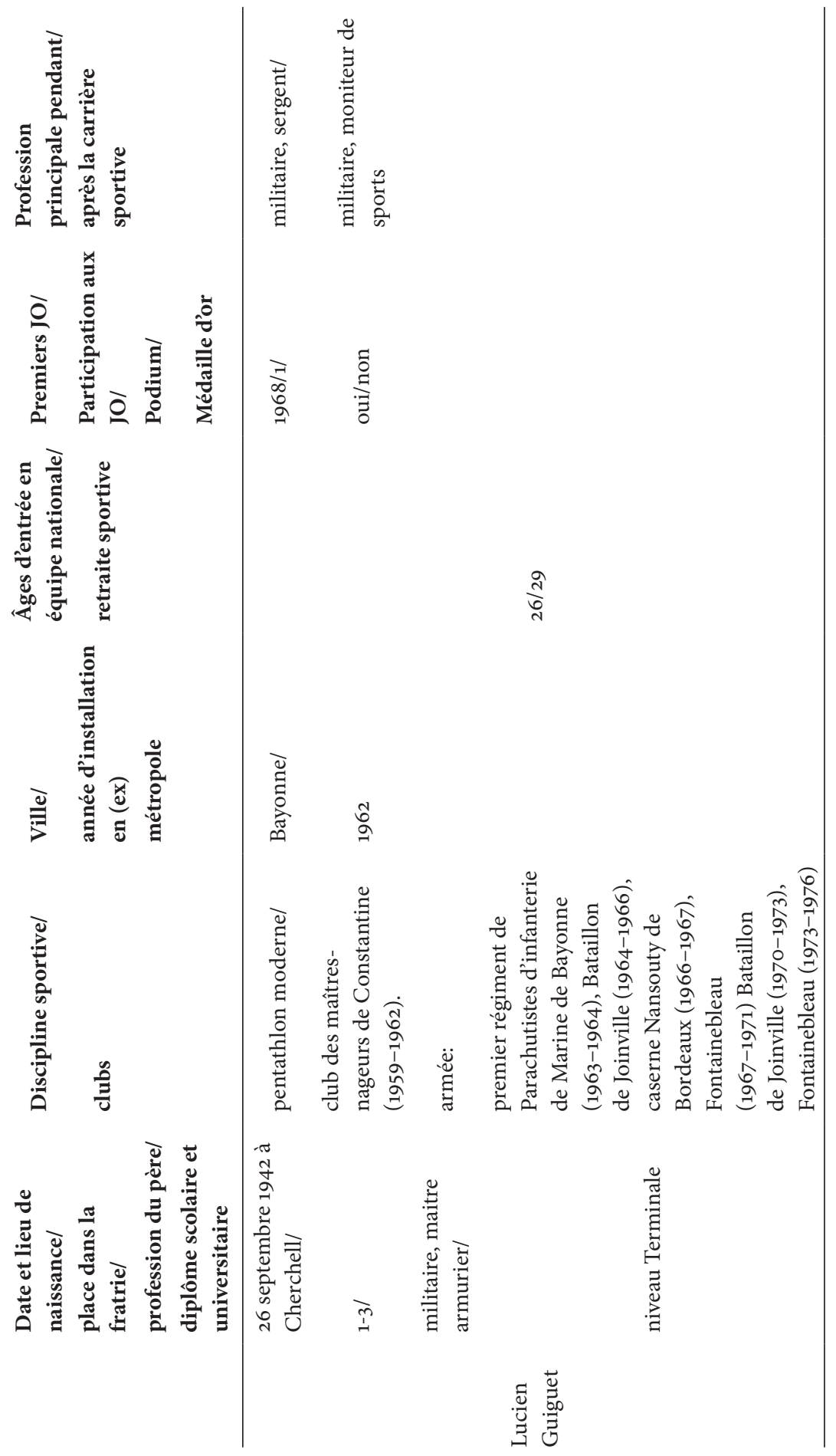




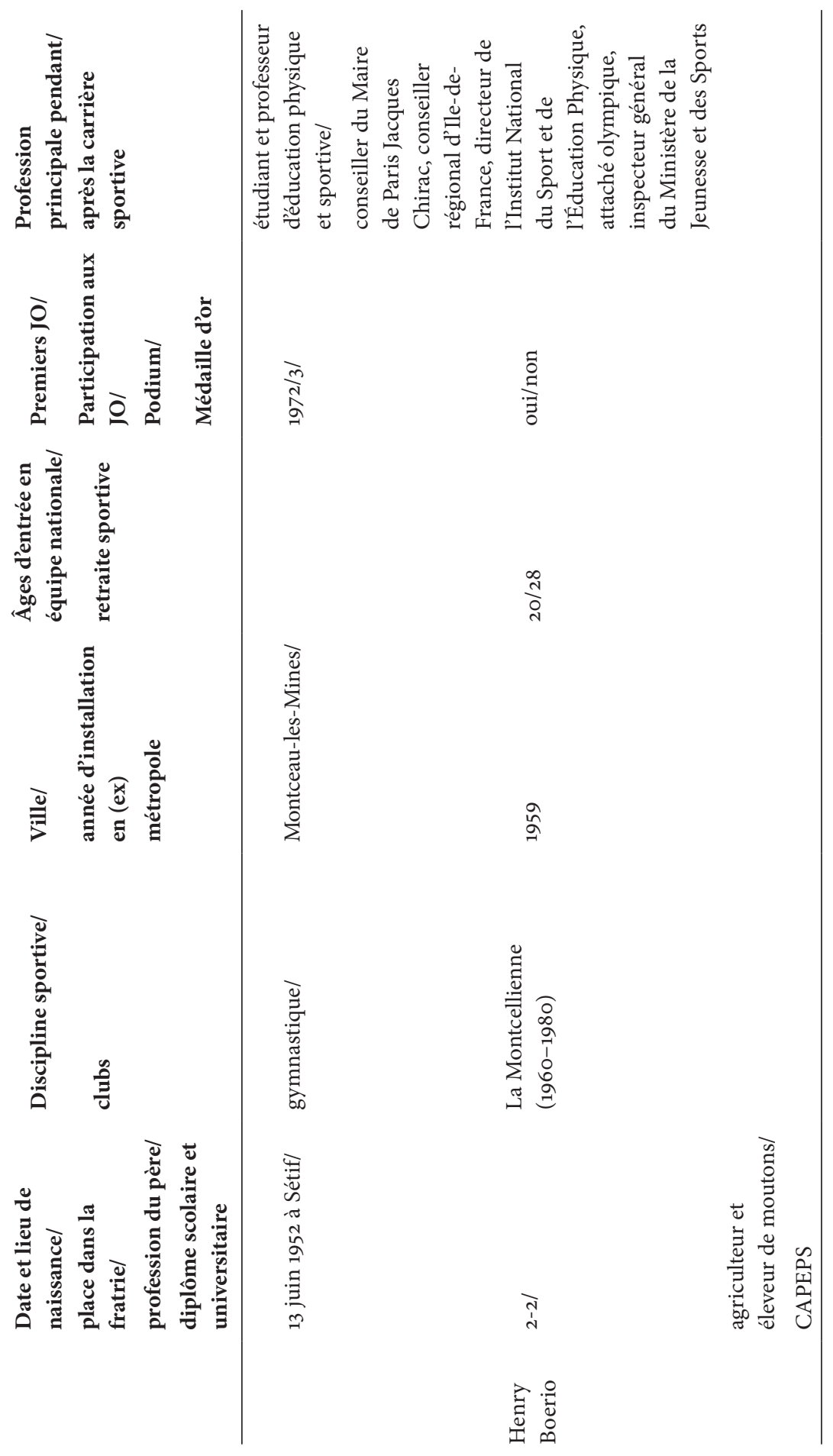




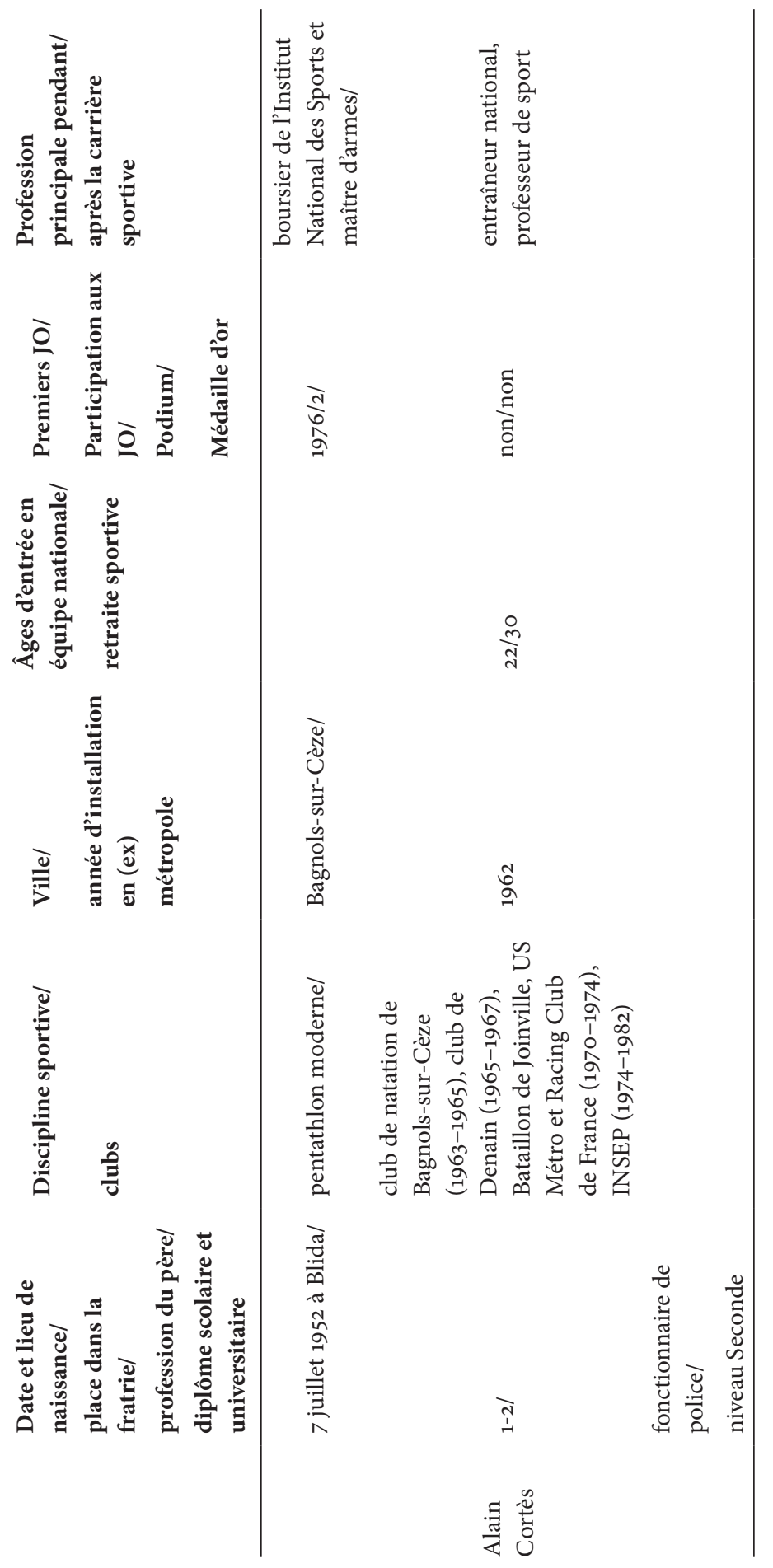




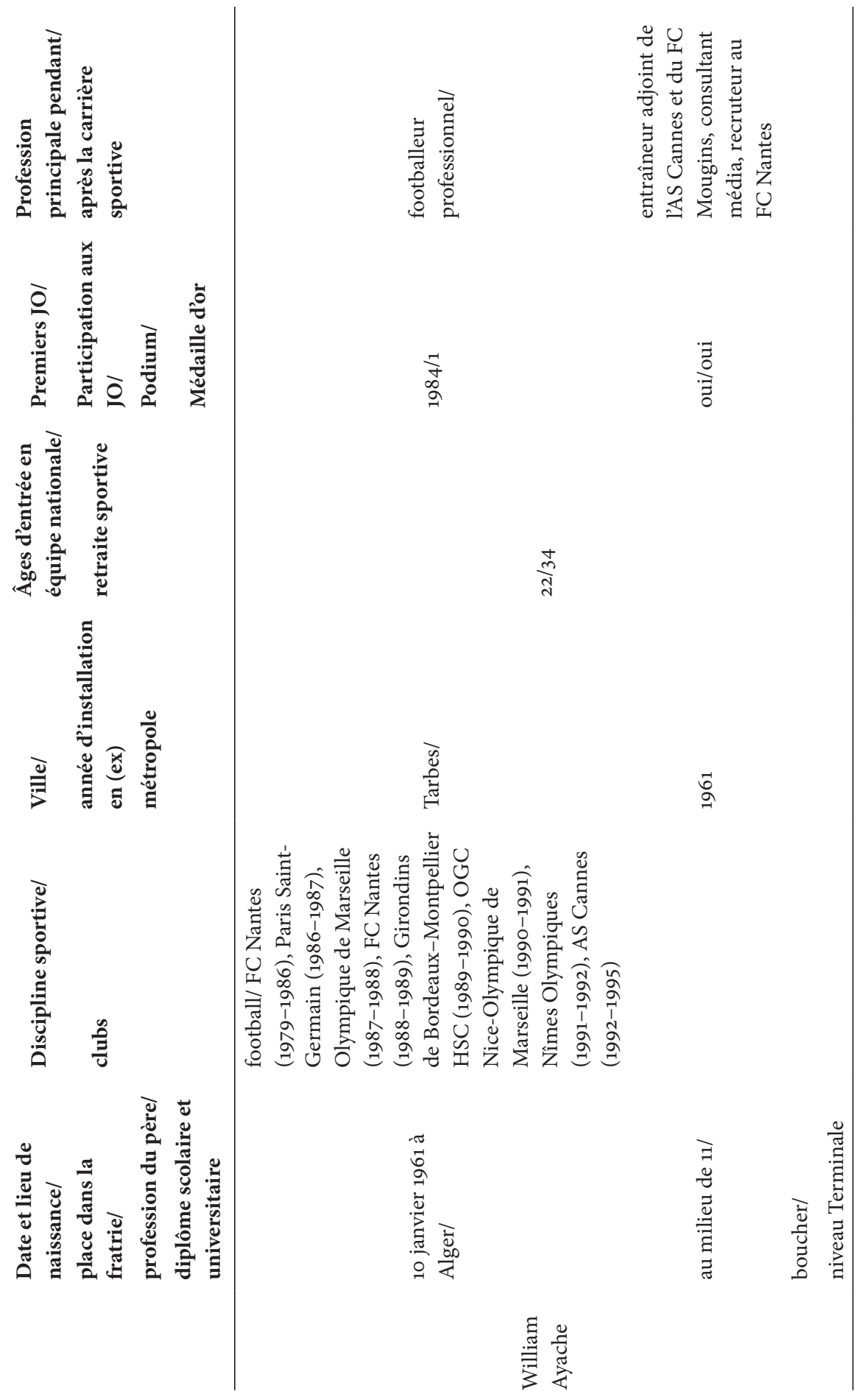




\section{BIBLIOGRAPHIE}

Archambault, Fabien. "Alger Basket, une communauté sportive en guerres (1939-1962)."

In L'aventure des grands hommes: études sur l'histoire du basket-ball, ed. Fabien Archambault, Loïc Artiaga et Pierre-Yves Frey, 167-171. Limoges: Pulim, 2004.

Arnaud, Pierre, et James Riordan. Sport et relations internationales: les démocraties face au fascisme et au nazisme (1900-1941). Paris: L'Harmattan, 1998.

Auvray Hernandez, Roland. Le livre d'or du football pied-noir et nord-africain. Toulon: Presses du Midi, 1995.

Ballester, Pierre. Michel Acariès, pied-noir, poings nus. De Bal-el-Oued à Las Vegas. Paris: Flammarion, 2018.

Bancel, Nicolas, Thomas Riot et Stanislas Frenkiel. Sports in Postcolonial Worlds (Abingdon, UK: Routledge, 2016).

Bancel, Nicolas, et Jean-Marc Gayman. Du guerrier à l'athlète: éléments d'histoire des pratiques corporelles. Paris: Presses Universitaires de France, 2002.

Bancel, Nicolas, Daniel Denis et Youssef Fatès. De l'Indochine à l'Algérie: la jeunesse en mouvement des deux côtés du miroir colonial (1940-1962). Paris: La Découverte, 2003.

Baussant, Michèle. "Exils et construction de la mémoire généalogique: l’exemple des piedsnoirs." Pôle Sud 1, no. 24 (2006): 29-44.

Berger, Peter L., et Thomas Luckmann. La construction sociale de la réalité. Paris: Armand Colin, 1966.

Bertaux, Daniel. Les récits de vie. Paris: Nathan, 1997.

Berthoud, Jérôme. Devenir, être et avoir été un footballeur camerounais. Thèse en Sociologie, Université de Lausanne, 2017.

Bertrand, Julien. La fabrique des footballeurs. Paris: La Dispute, 2012.

Bessol Lahouari, Ahmed. Foot algérien: les 100 étoiles (1954-2003). Alger: Entreprise algérienne de presse, 2003.

Bourdieu, Pierre. "L'illusion biographique." Actes de la Recherche en Sciences Sociales, no. $62-63$ (1986): 69-72.

Brissonneau, Christophe, Olivier Aubel et Fabien Ohl. Lépreuve du dopage: sociologie du cyclisme professionnel. Paris: Presses Universitaires de France, 2008.

Brittain, Ian. The Paralympic Games Explained. London: Routledge, 2010.

Brohm, Jean-Marie. Sociologie politique du sport. Paris: Delarge, 1976.

Callède, Jean-Paul. "Les politiques du sport en France." Lannée sociologique 52, no. 2 (2002): $437-457$ 
Chappelet, Jean-Loup, et Brenda Kübbler-Mabbott. The International Olympic Committee and the Olympic System: The Governance of World Sport. London: Routledge, 2008.

Chehat, Fayçal. La fabuleuse histoire du sport algérien. Alger: Al Bayazin, 2015.

Cheniki, Ahmed. "Algérie-France: expériences culturelles et aventures ambiguës." Hommes et Migrations, no. 1298 (2012): 82-94.

Chevally, Claude. Christian Lopez: du tac au tacle. Paris: Calmann-Lévy, 1970.

Chovaux, Olivier. "Introduction." Historiens et Géographes, no. 437 (2016): 35-36.

Clastres, Patrick. Jeux Olympiques: un siècle de passions. Paris: Musée National du Sport, 2008.

Coenen-Huther, Jacques. Sociologie des élites. Paris: Armand Colin, 2004.

Comtat, Emmanuelle. "La question du vote pied-noir." Pôle Sud 1, no. 24 (2006): 75-88.

Corbin, Alain, Jean-Jacques Courtine et Georges Vigarello. Histoire du corps: les mutations du regard, le XX $X^{\text {eme }}$ siècle. Paris: Points, 2006.

Corinne, Bertrand. Volley-ball raconté par Alain Fabiani. Paris: Hatier Rageot, 1986.

Couto, Marie-Paule. "Lintégration socio-économique des pieds-noirs en France métropolitaine: le lien de citoyenneté à lépreuve." Revue européenne des migrations internationales 29, no. 3 (2013): 93-119.

Delaplace, Jean-Michel. Histoire du sport, histoire des sportifs. Paris: L'Harmattan, 2005.

Dietschy, Paul. "Le football africain, entre domination coloniale et émancipation." In L'Empire des sports: une histoire de la mondialisation culturelle, ed. Pierre Singaravélou et Julien Sorez, 53-69. Paris: Belin, 2010.

Dietschy, Paul, et Patrick Clastres. Sport, société et culture en France du XIX ${ }^{\text {ème }}$ siècle à nos jours. Paris: Hachette, 2006.

Dine, Philip. "Dresser la carte sportive de 'l'Algérie française': vitesse technologique et appropriation de l'espace." In L'Empire des sports: une histoire de la mondialisation culturelle, ed. Pierre Singaravélou et Julien Sorez, 105-116. Paris: Belin, 2010.

Dine, Philip, et Didier Rey. "Le football en guerre d’Algérie." Matériaux pour l'histoire de notre temps 2, no. 106 (2012): 27-32.

Dosse, François. Le pari biographique. Paris: La Découverte, 2005.

Duret, Pascal. L'héroïsme sportif. Paris: Presses Universitaires de France, 1993.

Eldridge, Claire. "Returning to the 'Return': Pied-noir Memories of 1962." Revue européenne des migrations internationales 29, no. 3 (2013): 121-140.

Escanglon-Morin, Valérie. "La mémoire déchirée des pieds-noirs." Hommes et Migrations, no. 1251 (2004): 99-109.

Falcoz, Marc, et Michel Koebel. L'intégration par le sport: représentations et réalités. Paris: L'Harmattan, 2005. 
Fatès, Youssef. "Algérie coloniale: les patronages et le sport." In Sports et loisirs dans les colonies XIX ${ }^{\text {ème }}-X X^{\text {ème }}$ siècles, ed. Évelyne Combeau-Mari, 203-218. Paris: Le Publieur, 2004.

Fatès, Youssef. "Le club sportif, structure d'encadrement et de formation nationaliste de le jeunesse musulmane pendant la période coloniale." In De l'Indochine à l'Algérie: la jeunesse en mouvement des deux côtés du miroir colonial (1940-1962), ed. Nicolas Bancel, Daniel Denis et Youssef Fatès, 150-162. Paris: La Découverte, 2003.

Fatès, Youssef. Sport et politique en Algérie, de la période coloniale à nos jours. Thèse en Sciences politiques, Université Paris I, 2002.

Fatès, Youssef. Sport et Tiers-Monde. Paris: Presses Universitaires de France, 1994. Ferracci, Jean-Baptiste. L’adieu, 1962: le tragique exode des Français d’Algérie. Paris: Éditions de Paris, 2012.

Fleuriel, Sébastien, et Manuel Schotté. "Dépasser l'alternative amateurs/professionnels.” Le Mouvement Social, no. 254 (2016): 3-12.

Fleuriel, Sébastien, et Manuel Schotté. "La reconversion paradoxale des sportifs français: premiers enseignements d'une enquête sur les sélectionnés aux Jeux Olympiques de 1972 et 1992." Sciences sociales et sport, no. 4 (2011): 115-140.

Frenkiel, Stanislas. Le football des immigrés: France, Algérie, l'histoire en partage. Arras: Artois Presses Université, sous presse.

Frenkiel, Stanislas. "Larbi Ben Barek, Marcel Cerdan et Alfred Nakache: icônes de l'utopie impériale dans la presse métropolitaine (1936-1944)?" Staps 2, no. 80 (2008): 99-113.

Frenkiel, Stanislas, Julie Cornaton et Nicolas Bancel. "Les kings de l'athlétisme handisport: éléments pour la prosopographie d'une élite paralympique (1964-2014)." Social History 49, no. 100 (2016): 625-651.

Frenkiel, Stanislas, et Nicolas Penin. "Les footballeurs roubaisiens délite: trajectoires professionnelles et intégration sociale des années 1930 à nos jours." Revue du Nord 10o, no. 425 (2019): 337-368.

Gasparini, William. "Les champions des cités: parcours migratoires et effets de quartier." Hommes et Migrations, no. 1285 (2010): 108-123.

Gay-Lescot, Jean-Louis. Sport et éducation sous Vichy (1940-1944). Lyon: Presses Universitaires de Lyon, 1991.

Guiot, Pascaline, et Fabien Ohl. "La reconversion des sportifs: une épreuve de la petitesse?" Loisir et société, no. 30 (2008): 385-416.

Guttmann, Allen. The Olympics: A History of the Modern Games. Urbana: University of Illinois Press, 2002. 
Héas, Stéphane. Les discriminations dans les sports. Nancy: Presses Universitaires de Nancy, 2010.

Heck, Sandra. "La France et les premières heures du pentathlon moderne olympique." Staps 4, no. 106 (2014): 71-86.

Holt, Richard, Pierre Lanfranchi et J. A. Mangan. European Heroes, Myth, Identity, Sport. London: Franck Cass, 1996.

Howe, David. The Cultural Politics of the Paralympic Movement: Through an Anthropological Lens. London: Routledge, 2008.

Hureau, Joëlle. La mémoire des pieds-noirs. Paris: Perrin, 2010.

Javerlhiac, Sophie. La reconversion des sportifs de haut-niveau: pouvoir et vouloir se former. Rennes: Presses Universitaires de Rennes, 2014.

Jéquier, François, Pierre Milza et Philippe Tétart. Le pouvoir des anneaux: les Jeux Olympiques à la lumière de la politique (1896-2004). Paris: Vuibert, 2004.

Lanfranchi, Pierre, et Alfred Wahl. Les footballeurs professionnels des années 1930 à nos jours. Paris: Hachette, 1995.

Le Noé, Olivier. "Marceau Crespin a-t-il existé? Éclairages sur une éclipse historiographique de l'analyse des politiques gaullistes du sport." Sciences Sociales et sport 1, no. 7 (2014): $11-41$.

Lions, Bernard. Jean-Francois Larios: j’ai joué avec le feu. Paris: Solar, 2017.

Liotard, Philippe. Le sport dans les sixties. Reims: Presses Universitaires de Reims, 2016.

Manchon, Michaël. Le Racing Universitaire d'Alger: un club sportif universitaire en milieu colonial (1927-1962). Calvisson: Jacques Gandini, 2005.

Mauger, Gérard, Claude Poliak et Bernard Pudal. Histoire des lecteurs. Paris: Nathan, 1999.

Meynier, Gilbert. Histoire intérieure du FLN (1954-1962). Paris: Fayard, 2002.

Moumen, Abderrahman. "De l'Algérie à la France: les conditions de départ et d'accueil des rapatriés, pieds-noirs et harkis en 1962." Matériaux pour l'histoire de notre temps 3 , no. 99 (2010): 60-68.

Nuytens, Williams. L'épreuve du terrain. Rennes: Presses Universitaires de Rennes, 2011. Ollivier, Jean-Paul. Roger Tessier: jétais le gorille du Général (1947-1970). Paris: Perrin, 2002. Pas, Niek. "Vélocemen, hiverneurs et Algériens: cyclisme et sociabilité sportive en Algérie (1885-1914)." Vingtième Siècle 4, no. 136 (2017): 3-19.

Paugam, Serge. Le lien social. Paris: Presses Universitaires de France, 2008.

Pervillé, Guy. "L'abandon des harkis." In L’Algérie des Français, ed. Charles-Robert Ageron, 303-312. Paris: Le Seuil, 1993.

Pervillé, Guy. "Le terrorisme dans la Guerre d’Algérie." In L’Algérie des Français, ed. CharlesRobert Ageron, 207-222. Paris: Le Seuil, 1993. 
Rey, Didier. Football et société en Oranie coloniale (1897-1962). Mémoire d'Habilitation à Diriger des Recherches. Università di Corsica, 2010.

Rey, Didier. "Les identités multiples: Corse et Algérie au miroir du football (1897-1962)." Insaniyat, no. 34 (2006): 29-45.

Ripoll, Hubert. La résilience par le sport. Paris: Odile Jacob, 2016.

Rouet, Christophe. Les pieds-noirs: lépopée d’un peuple. Clermont-Ferrand: De Borée, 2016.

Rousso, Henry. "Les raisins verts de la guerre d'Algérie." In La Guerre d'Algérie (1954-1962), ed. Yves Michaud, 127-151. Paris: Odile Jacob, 2004.

Savarèse, Éric. "Mobilisations politiques et posture victimaire chez les militants associatifs pieds-noirs." Raisons politiques, no. 30 (2008): 41-58.

Scioldo-Zürcher, Yann. "Faire des Français d’Algérie des métropolitains." Pôle Sud 1, no. 24 (2006): 15-28.

Soula, Denis. Le Bataillon de Joinville: l'extraordinaire histoire de l'armée des champions. Paris: Cherche Midi, 2016.

Stora, Benjamin. La gangrène et l'oubli: la mémoire de la Guerre d'Algérie. Paris: La Découverte, 1991.

Stora, Benjamin. Histoire de la Guerre d'Algérie (1954-1962). Paris: La Découverte, 1993.

Terret, Thierry. Histoire du sport. Paris: Presses Universitaires de France, 2007.

Terret, Thierry, Luc Robène, Pascal Charroin, Stéphane Héas et Philippe Liotard. Sport, genre et vulnérabilité au XXème siècle. Rennes: Presses Universitaires de Rennes, 2013. Verdès-Leroux, Jeannine. "Les Français d’Algérie de 1830 à aujourd'hui." In La Guerre d’Algérie (1954-1962), ed. Yves Michaud, 63-85. Paris: Odile Jacob, 2004.

Villepreux, Olivier, Samy Mouhoubi et Frédéric Bernard. "1944, Occuper le terrain: Alexandre Villaplana, France." In Débordements: sombres histoires du football (1938-2016), ed. Olivier Villepreux, Samy Mouhoubi et Frédéric Bernard, 29-57. Paris: Anamosa, 2016.

Wallenborn, Hélène. L'historien, la parole des gens et l'écriture de l'histoire. Gilly: Labor, 2006. Winock, Michel. "La France et l'Algérie: 130 ans d'aveuglement." In La Guerre d'Algérie (1954-1962), ed. Yves Michaud, 9-28. Paris: Odile Jacob, 2004.

Zakine, Hubert. La mémoire du football d'Afrique du Nord. Murcia: Jimenez Godoy, 1995.

\section{NOTES}

1. Valérie Escanglon-Morin, "La mémoire déchirée des pieds-noirs," Hommes et Migrations, no. 1251 (2004): 99. La présente recherche est réalisée grâce au soutien 
institutionnel et financier de l'Académie Nationale Olympique Française via la bourse du Centre d'Études Olympiques Français. Si dans cet article le masculin est le plus souvent employé, l'auteur souhaite remercier toutes les sportives et tous les sportifs interviewés. Ils l’autorisent à citer leurs propos.

2. Joëlle Hureau, La mémoire des pieds-noirs (Paris: Perrin, 2010), 13.

3. Abderrahman Moumen, "De l'Algérie à la France, les conditions de départ et d'accueil des rapatriés, pieds-noirs et harkis en 1962," Matériaux pour l'histoire de notre temps 3 , no. 99 (2010): 60-68; Emmanuelle Comtat, "La question du vote pieds-noirs," Pôle Sud 1, no. 24 (2006): 75-88; Claire Eldridge, "Returning to the 'Return': Pied-noir Memories of 1962," Revue européenne des migrations internationales 29, no. 3 (2013): 121-140.

4. Michel Winock, "La France et l'Algérie: 130 ans d'aveuglement," in La Guerre d'Algérie (1954-1962), ed. Yves Michaud (Paris: Odile Jacob, 2004), 11.

5. Winock, "La France et l'Algérie: 130 ans d'aveuglement," 18.

6. Jeannine Verdès-Leroux, "Les Français d'Algérie de 1830 à aujourd'hui," in La Guerre d’Algérie (1954-1962), ed. Yves Michaud (Paris: Odile Jacob, 2004), 63-85.

7. "Lélite est traitée tantôt comme une pure catégorie de stratification sociale, tantôt comme une minorité consciente de ses valeurs et de ses intérêts, tantôt comme un synonyme euphémique de 'classe dominante' [ . . . ] Ce sont les élites du sport-c'est-à-dire les sportifs de haut-niveau-qui se laissent le plus facilement repérer sur la base exclusive de leurs performances"; Jacques Coenen-Huther, Sociologie des élites (Paris: Armand Colin, 2004), 3-106.

8. Nicolas Bancel, Thomas Riot et Stanislas Frenkiel, Sports in Postcolonial Worlds (Abingdon, UK: Routledge, 2016).

9. Nicolas Bancel et Jean-Marc Gayman, Du guerrier à l'athlète: éléments d'histoire des pratiques corporelles (Paris: Presses Universitaires de France, 2002).

10. Olivier Chovaux, "Introduction," Historiens et Géographes, no. 437 (2016): 35-36.

11. Paul Dietschy et Patrick Clastres, Sport, société et culture en France du XIX'me siècle à nos jours (Paris: Hachette, 2006).

12. Pierre Arnaud et James Riordan, Sport et relations internationales: les démocraties face au fascisme et au nazisme (1900-1941) (Paris: L'Harmattan, 1998).

13. Stéphane Héas, Les discriminations dans les sports (Nancy: Presses Universitaires de Nancy, 2010).

14. Christophe Brissonneau, Olivier Aubel et Fabien Ohl, Leépreuve du dopage: sociologie du cyclisme professionnel (Paris: Presses Universitaires de France, 2008). 
15. Jean-Michel Delaplace, Histoire du sport, histoire des sportifs (Paris: L'Harmattan, 2005).

16. Williams Nuytens, Lépreuve du terrain (Rennes: Presses Universitaires de Rennes, 2011).

17. Jean-Marie Brohm, Sociologie politique du sport (Paris: Delarge, 1976).

18. Pascal Duret, L'héroïsme sportif (Paris: Presses Universitaires de France, 1993); Richard Holt, Pierre Lanfranchi et J. A. Mangan, European Heroes, Myth, Identity, Sport (London: Franck Cass, 1996).

19. Allen Guttmann, The Olympics: A History of the Modern Games (Urbana: University of Illinois Press, 2002); Patrick Clastres, Jeux Olympiques: un siècle de passions (Paris: Musée National du Sport, 2008).

20. François Jéquier, Pierre Milza et Philippe Tétart, Le pouvoir des anneaux: les Jeux Olympiques à la lumière de la politique (1896-2004) (Paris: Vuibert, 2004).

21. Sandra Heck, "La France et les premières heures du pentathlon moderne olympique," Staps 4, no. 106 (2014): 71-86.

22. Jean-Loup Chappelet et Brenda Kübbler-Mabbott, The International Olympic Committee and the Olympic System: The Governance of World Sport (London: Routledge, 2008).

23. Alain Corbin, Jean-Jacques Courtine et Georges Vigarello, Histoire du corps: les mutations du regard, le XX'me siècle (Paris: Points, 2006); David Howe, The Cultural Politics of the Paralympic Movement: Through an Anthropological Lens (London: Routledge, 2008); Ian Brittain, The Paralympic Games Explained (London: Routledge, 2010); Stanislas Frenkiel, Julie Cornaton et Nicolas Bancel, "Les kings de lathlétisme handisport: éléments pour la prosopographie d'une élite paralympique (1964-2014)," Social History 49, no. 100 (2016): 625-651.

24. Youssef Fatès, Sport et politique en Algérie, de la période coloniale à nos jours, Thèse en Sciences politiques (Université Paris I, 2002); Didier Rey, Football et société en Oranie coloniale (1897-1962), Mémoire d'Habilitation à Diriger des Recherches (Università di Corsica, 2010).

25. Fabien Archambault, "Alger Basket, une communauté sportive en guerres (1939-1962)," in L'aventure des grands hommes: études sur l'histoire du Basket-Ball, ed. Fabien Archambault, Loïc Artiaga et Pierre-Yves Frey (Limoges: Pulim, 2004), 167-171; Philip Dine, "Dresser la carte sportive de l'Algérie française': vitesse technologique et appropriation de l'espace," in L'Empire des sports: une histoire de la mondialisation culturelle, ed. Pierre Singaravélou et Julien Sorez (Paris: Belin, 
2010), 105-116; Niek Pas, "Vélocemen, hiverneurs et Algériens: cyclisme et sociabilité sportive en Algérie (1885-1914)," Vingtième Siècle 4, no. 136 (2017): 3-19.

26. Roland Auvray Hernandez, Le livre d'or du football pied-noir et nord-africain (Toulon: Presses du Midi, 1995); Hubert Zakine, La mémoire du football d'Afrique du Nord (Murcia: Jimenez Godoy, 1995); Ahmed Bessol Lahouari, Foot algérien: les 100 étoiles (1954-2003) (Alger: Entreprise algérienne de presse, 2003); Fayçal Chehat, La fabuleuse histoire du sport algérien (Alger: Al Bayazin, 2015).

27. S’ajoutent à eux deux autres sportifs nés en métropole pour des raisons conjoncturelles mais ayant passé leur enfance à Alger où se trouvent leurs racines familiales. Il s’agit de l’escrimeur Jean-Claude Magnan, né à Aubagne en 1941, et du pentathlète Jean-Pierre Giudicelli, né à Pau en 1943.

28. Une trajectoire biographique peut être définie comme "un ensemble de parcours simultanés ou successifs dans divers cadres institutionnels, dans différents champs de l'espace social qui sont eux-mêmes en perpétuel changement"; Gérard Mauger, Claude Poliak et Bernard Pudal, Histoire des lecteurs (Paris: Nathan, 1999), 13. Lié à l'enchevêtrement d'une histoire familiale, d'un cursus scolaire, d'une carrière professionnelle, d'un itinéraire politique et religieux, ce concept de "trajectoire" apparaît comme plus fécond que celui de "carrière," cantonné ici au secteur professionnel et à un seul cheminement sectoriel.

29. Marc Falcoz et Michel Koebel, Lintégration par le sport: représentations et réalités (Paris: L'Harmattan, 2005).

30. Sébastien Fleuriel et Manuel Schotté, "La reconversion paradoxale des sportifs français: premiers enseignements d'une enquête sur les sélectionnés aux Jeux Olympiques de 1972 et 1992," Sciences sociales et sport, no. 4 (2011): 115-140.

31. Tous sont sélectionnés aux Jeux Olympiques (date de naissance, première participation olympique, discipline sportive): Paul Masson (1876, 1896, cyclisme), Serge Dastarac (1888, 1908, football), Georges Verger (1896, 1924, athlétisme), Charles Bardot (1904, 1928, athlétisme), Alexandre Villaplane (1905, 1928, football), Jacques Wild (1905, 1928, football), Alfred Nakache (1915, 1936, natation), René Aleman (1913, 1948, haltérophilie), Joseph Bernardo (1929, 1948, natation), Patrick El Mabrouk (1928, 1952, athlétisme), René Million (1934, 1952, natation), Maryse Morandini (1932, 1952, natation), Armand Mouyal (1925, 1952, escrime), Célestin Oliver (1930, 1952, football), Jean-Pierre Salignon (1928, 1952, basket-ball), Héda Frost (1936, 1956, natation), Guy Monserret (1935, 1956, natation), Simone Brierre (1937, 1960, athlétisme), Robert Caymaris (1935, 1960, gymnastique), Jean-Pascal Curtillet (1942, 1960, natation), André Giamarchi (1931, 1960, football), Marc Kamoun (1937, 
1960, natation), Jean-Claude Magnan (1941, 1960, escrime), Françoise Borie (1947, 1964, natation), Alain Gotvallès (1942, 1964, natation), Monique Pietri (1945, 1964, natation), Jean-Paul Anton (1943, 1968, boxe), Jean-Pierre Giudicelli (1943, 1968, pentathlon moderne), Lucien Guiguet (1942, 1968, pentathlon moderne), Pierre Amardeilh (1951, 1972, natation), Henry Boerio (1952, 1972, gymnastique), Jacques Filippini (1950, 1972, aviron), Chantal Schertz (1958, 1972, natation), Jacques Aletti (1955, 1976, athlétisme), Pierre Andraca (1958, 1976, natation), Alain Cortès (1952, 1976, pentathlon moderne), Jean Fernandez (1954, 1976, football), Dominique Petit (1961, 1980, natation), Michel Salesse (1955, 1980, escrime), William Ayache (1961, 1984, football), Franck Ducheix (1962, 1984, escrime), Michel Nowak (1962, 1984, judo), Patrick Birocheau (1955, 1988, tennis de table), Alain Fabiani (1958, 1988, volleyball), Pierre Garsau (1961, 1988, water-polo), Odile Barré (1962, 1992, voile) et Frédéric Perez (1961, 1992, handball).

32. Il est construit sur une soixantaine de questions ouvertes regroupées en trois grands thèmes: la vie durant l'enfance, l'accès au sport et au haut-niveau (l'origine sociale et culturelle et trajets vers le sport et l'équipe nationale); l'expérience olympique (les Jeux sous le maillot bleu et la transformation d'une discipline); l'excellence sportive, migration et identité (les conditions du départ et d’accueil, les réseaux, l'arrêt de la carrière, la transition et la reconversion sportive).

33. François Dosse, Le pari biographique (Paris: La Découverte, 2005).

34. Daniel Bertaux, Les récits de vie (Paris: Nathan, 1997), 33.

35. Hélène Wallenborn, L'historien, la parole des gens et l'écriture de l'histoire (Gilly: Labor, 2006).

36. Pierre Bourdieu, "Lillusion biographique," Actes de la Recherche en Sciences Sociales, no. 62-63 (1986): 69-72.

37. Jean-Louis Gay-Lescot, Sport et éducation sous Vichy (1940-1944) (Lyon: Presses Universitaires de Lyon, 1991).

38. Bertrand Corinne, Volley-ball raconté par Alain Fabiani (Paris: Hatier Rageot, 1986).

39. Bernard Lions, Jean-François Larios: j’ai joué avec le feu (Paris: Solar, 2017).

40. Claude Chevally, Christian Lopez: du tac au tacle (Paris: Calmann-Lévy, 1970).

41. Jean-Paul Ollivier, Roger Tessier: jétais le gorille du Général (1947-1970) (Paris: Perrin, 2002).

42. Pierre Ballester, Michel Acariès, pied-noir, poings nus. De Bal-el-Oued à Las Vegas (Paris: Flammarion, 2018).

43. Olivier Villepreux, Samy Mouhoubi et Frédéric Bernard, "1944, Occuper le terrain, Alexandre Villaplana, France," in Débordements: sombres histoires du football 
(1938-2016), ed. Olivier Villepreux, Samy Mouhoubi et Frédéric Bernard (Paris: Anamosa, 2016), 29-57.

44. Françoise Inizan, "L'intarissable Bernardo," L'Équipe Magazine, 30 juillet 2016: 23-25.

45. Il est accessible en intégralité sur le site: http://algerazur.canalblog.com/archives/2013/o2/28/26533659.html.

46. Éric Savarèse, "Mobilisations politiques et posture victimaire chez les militants associatifs pieds-noirs," Raisons politiques, no. 30 (2008): 41-58.

47. Henry Rousso, "Les raisins verts de la guerre d'Algérie," in La Guerre d'Algérie (1954-1962), ed. Yves Michaud (Paris: Odile Jacob, 2004), 127-151.

48. Thierry Terret, Histoire du sport (Paris: Presses Universitaires de France, 2007).

49. Bancel et Gayman, Du guerrier à l'athlète: éléments d'histoire des pratiques corporelles.

50. Rey, Football et société en Oranie coloniale (1897-1962).

51. Dine et Rey, "Le football en guerre d'Algérie."

52. Youssef Fatès, "Algérie coloniale: les patronages et le sport," in Sports et loisirs dans les colonies XIX'ème-XXème siècles, ed. Évelyne Combeau-Mari (Paris: Le Publieur, 2004), 203-218.

53. Paul Dietschy, "Le football africain, entre domination coloniale et émancipation," in L'Empire des sports, une histoire de la mondialisation culturelle, ed. Pierre Singaravélou et Julien Sorez (Paris: Belin, 2010), 53-69.

54. Ahmed Cheniki, "Algérie-France: expériences culturelles et aventures ambiguës," Hommes et Migrations, no. 1298 (2012): 82-94.

55. Youssef Fatès, Sport et Tiers-Monde (Paris: Presses Universitaires de France, 1994).

56. Youssef Fatès, "Le club sportif, structure d'encadrement et de formation nationaliste de le jeunesse musulmane pendant la période coloniale," in De l'Indochine à l'Algérie: la jeunesse en mouvement des deux côtés du miroir colonial (1940-1962), ed. Nicolas Bancel, Daniel Denis et Youssef Fatès (Paris: La Découverte, 2003), 150-162.

57. Stanislas Frenkiel, "Larbi Ben Barek, Marcel Cerdan et Alfred Nakache: icônes de l’utopie impériale dans la presse métropolitaine (1936-1944)?” Staps 2, no. 80 (2008): 99-113.

58. Dans la nuit du 8 au 9 septembre 1954, de retour du cinéma, père et fils font partie des sinistrés du tremblement de terre qui ravage la région d'Orléansville. Les secours dénombrent plus de 1300 morts et 5 ooo blessés.

59. Joseph Bernardo, Marseille, 14 février 2019.

6o. Fatès, Sport et politique en Algérie, de la période coloniale à nos jours.

61. Héda Frost, Aix-en-Provence, 12 février 2019. 
62. Michaël Manchon, Le Racing Universitaire d'Alger, un club sportif universitaire en milieu colonial (1927-1962) (Calvisson: Jacques Gandini, 2005).

63. Fatès, Sport et politique en Algérie, de la période coloniale à nos jours.

64. Dine et Rey, "Le football en guerre d’Algérie."

65. "L'Organisation de l'Armée Secrète est vraisemblablement créée au début de l'année 1961 par Pierre Lagaillarde, ancien avocat, député algérois et incarcéré suite à son implication à la tête du comité insurrectionnel de la semaine des barricades. En mai 1961 est constitué un 'comité directeur' de l'OAS et les liaisons sont établies avec les généraux Edmond Jouhaud et Raoul Salan à qui est attribué le commandement suprême. Ses objectifs sont simples: rester fidèle à l'esprit du 13 mai 1958, résister à la politique du 'dégagement' algérien menée par le pouvoir gaulliste, construire une Algérie nouvelle 'fraternelle et française.' L'OAS pratique la politique du pire et enchaine les actions terroristes avec la participation et la complicité de la population européenne. Le climat de violence s'exaspère mais au jeu du terrorisme et du contreterrorisme, l'OAS voit fondre ses très faibles effectifs combattants, et se radicaliser son action, notamment après les Accords d'Évian jusqu’en juin 1962 où les derniers commandos de l'OAS prennent le chemin de l'exil." Benjamin Stora, Histoire de la Guerre d’Algérie (1954-1962) (Paris: La Découverte, 1993), 60-61.

66. Jean-Paul Anton, entretien téléphonique, 30 janvier 2019.

67. Le FLN est une organisation indépendantiste qui revendique les spectaculaires opérations militaires du $1^{\text {er }}$ novembre 1954 et qui, au sortir de la Guerre d'Algérie, s'installe comme un parti unique niant tout pluralisme politique et culturel. Gilbert Meynier, Histoire intérieure du FLN (1954-1962) (Paris: Fayard, 2002).

68. Stora, Histoire de la Guerre d'Algérie (1954-1962).

69. Dine et Rey, "Le football en guerre d'Algérie."

70. "La première harka est constituée dans l'Aurès dès novembre 1954. Mais pendant deux ans, l'expérience est limitée par la crainte des désertions et des fuites d'armes. C'est le Général Salan qui décide d'intensifier le recrutement de soldats et de supplétifs musulmans: sous son commandement, leur total passe de 38 ooo hommes en janvier 1957 à 103000 en décembre 1958, dépassant ainsi largement l'effectif maximal atteint par l'Armée de Libération Nationale au début de 1958." Guy Pervillé, "Labandon des harkis," in L'Algérie des Français, ed. Charles-Robert Ageron (Paris: Le Seuil, 1993), 304.

71. Guy Pervillé, "Le terrorisme dans la Guerre d’Algérie," in L’Algérie des Français, ed. Charles-Robert Ageron (Paris: Le Seuil, 1993), 207-222. 
72. Nicolas Bancel, Daniel Denis et Youssef Fatès, De l'Indochine à l'Algérie: la jeunesse en mouvement des deux côtés du miroir colonial (1940-1962) (Paris: La Découverte, 2003).

73. Didier Rey, "Les identités multiples: Corse et Algérie au miroir du football (1897-1962)", Insaniyat, no. 34 (2006): 29-45.

74. Rey, Football et société en Oranie coloniale (1897-1962).

75. Jean-Baptiste Ferracci, L’adieu, 1962: le tragique exode des Français d'Algérie (Paris: Éditions de Paris, 2012).

76. Yann Scioldo-Zürcher, "Faire des Français d’Algérie des métropolitains," Pôle Sud 1, no. 24 (2006): 21.

77. Marc Kamoun, Rocquencourt, 15 janvier 2019.

78. Le Colonel Marceau Crespin occupe des responsabilités directoriales au sein de l'administration d'État des sports de 1961 à 1974. Ancien commandant de la base constantinoise d'Aïn-Arnat de 1955 à 1960, ce pilote d'hélicoptère et International militaire de rugby reste sensible à la cause des Français d'Algérie. Olivier Le Noé, "Marceau Crespin a-t-il existé? Éclairages sur une éclipse historiographique de l'analyse des politiques gaullistes du sport," Sciences Sociales et sport 1, no. 7 (2014): $11-41$.

79. Héda Frost, Aix-en-Provence, 12 février 2019.

8o. Lucien Guiguet, Marmande, 16 février 2019.

81. Stanislas Frenkiel, Le football des immigrés: France, Algérie, l'histoire en partage (Arras: Artois Presses Université, sous presse).

82. Françoise Borie, entretien téléphonique, 7 février 2019.

83. William Ayache, entretien téléphonique, 8 janvier 2008.

84. Hubert Ripoll, La résilience par le sport (Paris: Odile Jacob, 2016).

85. Philippe Liotard, Le sport dans les sixties (Reims: Presses Universitaires de Reims, 2016).

86. Joseph Bernardo, Marseille, 14 février 2019.

87. En décembre 1964, ce soutien volontariste s'inscrit dans l'Essai de doctrine du sport, approuvé par le Haut Comité des sports de Maurice Herzog en 1964, après plus d'une centaine de séances en deux années. Jean-Paul Callède, "Les politiques du sport en France," Lannée sociologique 52, no. 2 (2002): 437-457.

88. William Gasparini, "Les champions des cités, parcours migratoires et effets de quartier," Hommes et Migrations, no. 1285 (2010): 108-123.

89. "Les salaires sont multipliés par huit en 12 ans de 1978 à 1990. [ . . . L Les 'présidents vedettes', l'argent de la télévision, des maires et des publicités et sponsors en sont en partie responsables" écrivent les historiens Pierre Lanfranchi et Alfred Wahl. Pierre 
Lanfranchi et Alfred Wahl, Les footballeurs professionnels des années 1930 à nos jours (Paris: Hachette, 1995), 217-231.

90. Henry Boerio, Sainte-Maxime, 13 février 2019.

91. Julien Bertrand, La fabrique des footballeurs (Paris: La Dispute, 2012).

92. Denis Soula, Le Bataillon de Joinville: l'extraordinaire histoire de l'armée des champions (Paris: Cherche Midi, 2016).

93. Jean-Pierre Giudicelli, Venelles, 12 février 2019.

94. En 1977, l'Équipe de France de gymnastique, emmenée par Henry Boerio, fait une tournée de plusieurs semaines dans la Chine post-Mao Zedong. Elle est filmée par les caméras de TF1. Le reportage de 36 minutes est disponible sur le site de l'INA: https://www.ina.fr/video/CAA7701264001/le-sport-en-chine-video.html.

95. Inizan, "L'intarissable Bernardo," 24.

96. Héda Frost, Aix-en-Provence, 12 février 2019.

97. Marc Kamoun, Rocquencourt, 15 janvier 2019.

98. Lucien Guiguet, Marmande, 16 février 2019.

99. Jean-Pierre Giudicelli, Venelles, 12 février 2019.

100. Alain Cortès, entretien téléphonique, 29 janvier 2019.

101. Peter L. Berger et Thomas Luckmann, La construction sociale de la réalité (Paris: Armand Colin, 1966).

102. Pascaline Guiot et Fabien Ohl, "La reconversion des sportifs: une épreuve de la petitesse?" Loisir et société, no. 30 (2008): 385-416.

103. Fleuriel et Schotté, "La reconversion paradoxale des sportifs français: premiers enseignements d'une enquête sur les sélectionnés aux Jeux Olympiques de 1972 et 1992," 115-140.

104. Lanfranchi et Wahl, Les footballeurs professionnels des années 1930 à nos jours.

105. Sophie Javerlhiac, La reconversion des sportifs de haut-niveau: pouvoir et vouloir se former (Rennes: Presses Universitaires de Rennes, 2014); Jérôme Berthoud, Devenir, être et avoir été un footballeur camerounais, Thèse en Sociologie (Université de Lausanne, 2017); Stanislas Frenkiel et Nicolas Penin, "Les footballeurs roubaisiens d'élite, trajectoires professionnelles et intégration sociale des années 1930 à nos jours," Revue du Nord 100, no. 425 (2019): 337-368.

106. Ses intermèdes comme maître-nageur à l'Hôtel de Paris de Monaco et entraîneur de natation de l'AS Monaco ne lécartent pas de sa nouvelle et durable passion pour le théâtre.

107. Héda Frost, Aix-en-Provence, 12 février 2019. 
108. Christophe Rouet, Les pieds-noirs: lépopée d'un peuple (Clermont-Ferrand: De Borée, 2016).

109. Rassemblement et coordination unitaire des rapatriés et spoliés d’outre-mer.

110. Françoise Borie, entretien téléphonique, 7 février 2019.

111. Henry Boerio, Sainte-Maxime, 13 février 2019.

112. Hureau, La mémoire des pieds-noirs, 113-114.

113. Michèle Baussant, "Exils et construction de la mémoire généalogique: l’exemple des pieds-noirs," Pôle Sud 1, no. 24 (2006).

114. Verdès-Leroux, "Les Français d’Algérie de 1830 à aujourd'hui," 80.

115. Serge Paugam, Le lien social (Paris: Presses Universitaires de France, 2008).

116. Marie-Paule Couto, "L'intégration socio-économique des pieds-noirs en France métropolitaine: le lien de citoyenneté à lépreuve," Revue européenne des migrations internationales 29, no. 3 (2013): 93-119.

117. Benjamin Stora, La gangrène et l'oubli: la mémoire de la Guerre d'Algérie (Paris: La Découverte, 1991).

118. Thierry Terret, Luc Robène, Pascal Charroin, Stéphane Héas et Philippe Liotard, Sport, genre et vulnérabilité au XX ${ }^{\text {ème }}$ siècle (Rennes: Presses Universitaires de Rennes, 2013); Sébastien Fleuriel et Manuel Schotté, "Dépasser l’alternative amateurs/ professionnels," Le Mouvement Social, no. 254 (2016): 3-12.

119. Gay-Lescot, Sport et éducation sous Vichy (1940-1944).

120. Il s’agit de (année de première sélection): Pierre Chesneau (1924), Charles Bardot (1925), André Liminana (1925), Félix Pozo (1925), Georges Bonello (1926), Henri Salvano (1926), Alexandre Villaplane (1926), Jacques Wild (1927), Charles Allé (1929), Gustave Dubus (1930), Ernest Liberati (1930), Joseph Alcazar (1931), Joseph Rodriguez (1932), Max Charbit (1934), Émile Zermani (1935), Joseph Gonzales (1936), Michel Frutoso (1937), Emmanuel Aznar (1938), Jean Bastien (1938), Michel Brusseaux (1938), Mario Zatelli (1939), Lucien Jasseron (1945), Marcel Salva (1945), Jean-Claude Samuel (1945), Sauveur Rodriguez (1947), Manuel Garriga (1950), Célestin Oliver (1953), Georges Lamia (1959) et Bernard Rahis (1959). 